\title{
Predictions of the Nancy Grace Roman Space Telescope Galactic Exoplanet Survey. II. Free-floating Planet Detection Rates*
}

\author{
Samson A. Johnson ${ }^{1}\left(\mathbb{D}\right.$, Matthew Penny ${ }^{2}$ (D) B. Scott Gaudi ${ }^{1}$ (D), Eamonn Kerins ${ }^{3}$, Nicholas J. Rattenbury ${ }^{4}$ (i), Annie C. Robin ${ }^{5}$ (1), \\ Sebastiano Calchi Novati ${ }^{6}$, and Calen B. Henderson ${ }^{6}$ (i) \\ ${ }^{1}$ Department of Astronomy, The Ohio State University, 140 West 18th Avenue, Columbus, OH 43210, USA; johnson.7080@osu.edu \\ ${ }^{2}$ Department of Physics and Astronomy, Louisiana State University, Baton Rouge, LA 70803, USA \\ ${ }^{3}$ Jodrell Bank Centre for Astrophysics, Alan Turing Building, University of Manchester, Manchester M13 9PL, UK \\ ${ }^{4}$ Department of Physics, University of Auckland, Private Bag 92019, Auckland, New Zealand \\ ${ }^{5}$ Institut Utinam, CNRS UMR 6213, OSU THETA, Universite Bourgogne-Franche-Comt'e, 41bis avenue de l'Observatoire, F-25000 Besançon, France \\ ${ }^{6}$ IPAC, Mail Code 100-22, Caltech, 1200 East California Boulevard, Pasadena, CA 91125, USA \\ Received 2020 June 23; revised 2020 July 15; accepted 2020 July 16; published 2020 August 21
}

\begin{abstract}
The Nancy Grace Roman Space Telescope (Roman) will perform a Galactic Exoplanet Survey (RGES) to discover bound exoplanets with semimajor axes greater than 1 au using gravitational microlensing. Roman will even be sensitive to planetary-mass objects that are not gravitationally bound to any host star. Such free-floating planetarymass objects (FFPs) will be detected as isolated microlensing events with timescales shorter than a few days. A measurement of the abundance and mass function of FFPs is a powerful diagnostic of the formation and evolution of planetary systems, as well as the physics of the formation of isolated objects via direct collapse. We show that Roman will be sensitive to FFP lenses that have masses from that of Mars $\left(0.1 M_{\oplus}\right)$ to gas giants $\left(M \gtrsim 100 M_{\oplus}\right)$ as isolated lensing events with timescales from a few hours to several tens of days, respectively. We investigate the impact of the detection criteria on the survey, especially in the presence of finite-source effects for low-mass lenses. The number of detections will depend on the abundance of such FFPs as a function of mass, which is at present poorly constrained. Assuming that FFPs follow the fiducial mass function of cold, bound planets adapted from Cassan et al., we estimate that Roman will detect $\sim 250$ FFPs with masses down to that of Mars (including $\sim 60$ with masses $\leqslant M_{\oplus}$ ). We also predict that Roman will improve the upper limits on FFP populations by at least an order of magnitude compared to currently existing constraints.
\end{abstract}

Unified Astronomy Thesaurus concepts: Gravitational microlensing (672); Free floating planets (549); Space telescopes (1547); Exoplanet detection methods (489)

\section{Introduction}

Time and again, surprising results have arisen from searches for planets beyond our solar system. Indeed, one of the first planets discovered defined a population of "hot Jupiters" (e.g., Mayor \& Queloz 1995). These gas giant planets have orbital periods on the order of days and can have equilibrium temperatures hotter than many stars (Collier Cameron et al. 2010; Gaudi et al. 2017). The Kepler mission revealed a substantial population of "super-Earths" (Léger et al. 2009), planets with radii between those of Earth and Neptune-planets that have no analog in our solar system. Strange system architectures and planet hosts add even more variety, including planets in tightly packed systems (Gillon et al. 2017), planets orbiting both stars of a binary system (Doyle et al. 2011), planetary systems orbiting pulsars (Wolszczan \& Frail 1992), and planetary systems orbiting stars at the very bottom of the main sequence (Gillon et al. 2017). There appear to be almost no physical constraints on where exoplanets may reside.

Despite this diversity, our statistical census of exoplanets remains substantially incomplete. One area of parameter space that has yet to be fully explored is that of planetary-mass objects that are unbound from any host star. A population of free-floating planetary-mass objects (FFPs) in our Galaxy could have two primary sources. First, such bodies could be formed

\footnotetext{
* During the preparation of this manuscript, the name of the Wide Field Infrared Survey Telescope was changed to the Nancy Grace Roman Space Telescope.
}

in relative isolation. These would essentially be the lowestmass objects assembled through star formation processes. Second, such objects could form in a protoplanetary disk initially bound to a host star and later become liberated from their host. Regardless of their origin, we will refer to objects with masses comparable to planets that are not bound to any host as FFPs.

There are several mechanisms that could lead to the formation of isolated low-mass stellar objects (see Luhman 2012, and references therein). Stellar cores can be formed at a range of masses through either gravitational or turbulent compression and fragmentation (Bonnell et al. 2008). Here, the lowest-mass cores would result in the lowest-mass compact objects; this process may extend down to planetary-mass objects. Alternatively, the accretion of gas onto a protostellar core can be truncated, e.g., by being dynamically ejected from their birth clouds by other cores, or by radiation from nearby hot stars that photoevaporate the envelope from around the forming star (e.g., Bate 2009).

Photometric surveys of star-forming regions can constrain populations of such low-mass stellar objects (e.g., Gagné et al. 2017). These surveys are most sensitive to young objects that have not had time to radiate away their thermal energy from formation and thus remain luminous. In the field, the first 13 class-defining $\mathrm{Y}$ dwarfs were discovered by Cushing et al. (2011) and Kirkpatrick et al. (2012) using the Wide-field Infrared Survey Explorer (Wright et al. 2010). Modeled masses for these objects are of order tens of Jupiter masses $\left(M_{\text {Jup }}\right)$. 
Volume-limited searches for ultracool field dwarfs (e.g., Bardalez Gagliuffi et al. 2019) constrain these populations, but their low luminosities limit the number of detections and thus the statistical power of these surveys. Furthermore, these surveys are unlikely to be sensitive to planets with masses substantially smaller than that of Jupiter, regardless of their ages.

On the other hand, if the dominant reservoir of FFPs is a population of previously bound planets, there is no shortage of methods to liberate them from their hosts. Planets can be ejected from their systems by the chaotic processes that occur during planet formation (e.g., Rasio \& Ford 1996), be stripped from their stars by stellar flybys (e.g., Malmberg et al. 2011), or become unbound during the post-main-sequence evolution of their hosts (e.g., Adams et al. 2013). Hong et al. (2018) predict that planet-planet dynamical interactions could also eject lunarmass satellites of these planets during the encounters. It is important to emphasize that objects in the lowest-mass regime $\left(<1 M_{\mathrm{Jup}}\right)$ are very difficult to detect by any radiation they emit, even when they are young (Spiegel \& Burrows 2012).

A robust method to detect isolated planetary-mass objects is gravitational microlensing (Di Stefano \& Scalzo 1999). A microlensing event occurs when a massive body (the lens) passes in front of a background star (the source) within roughly one angular Einstein ring radius $\theta_{\mathrm{E}}$ of the lens,

$$
\theta_{\mathrm{E}}=\sqrt{\kappa M \pi_{\mathrm{rel}}} \text {. }
$$

Here $M$ is the mass of the lensing body, the constant $\kappa=4 G\left(c^{2} \mathrm{au}\right)^{-1}=8.14$ mas $M_{\odot}^{-1}$, and the lens-source relative parallax is $\pi_{\text {rel }}=1 \mathrm{au}\left(D_{\mathrm{L}}^{-1}-D_{\mathrm{S}}^{-1}\right)$, where $D_{\mathrm{L}}$ and $D_{\mathrm{S}}$ are the distances from the observer to the lens and source, respectively.

When the angular separation of the lens and source is comparable to or smaller than $\theta_{\mathrm{E}}$, the background source is significantly magnified. The duration of an event is characterized by the microlensing timescale $t_{\mathrm{E}}=\theta_{\mathrm{E}} / \mu_{\text {rel }}$. Thus, the size of the Einstein ring in combination with the lens-source relative proper motion $\left(\mu_{\text {rel }}\right)$ dictates the duration of the event, which can last from a few hours to a few hundred days, depending on the values of the above variables. The primary reason why microlensing is a powerful technique to detect FFPs is that it does not rely on the detection of any light from these essentially dark lenses.

While the phenomenology of typical microlensing events (for which $\theta_{\mathrm{E}}$ is much greater than the angular source size) is well understood, that of microlensing events due to low-mass objects has not been frequently discussed. We therefore include a short review of the phenomenology of low-mass microlensing (specifically when the angular source size is larger than $\theta_{\mathrm{E}}$ ) in Appendix A.

One of the pioneering uses of the technique was the search for the then-viable dark matter candidate massive compact halo objects, or MACHOs. At the time, the typical mass for these candidates for dark matter was unknown, resulting in the need to design a survey that was sensitive to the full range of timescales mentioned above. The major microlensing collaborations included the Expérience pour la Recherche d'Objets Sombres (EROS; Renault et al. 1997), the MACHO Collaboration (Alcock et al. 1997), the Microlensing Observations in Astrophysics Collaboration (MOA-I; Muraki et al. 1999), and the Optical Gravitation Lens Experiment (OGLE-I; Udalski et al. 1992). These collaborations set out to detect these MACHOs by monitoring the Large Magellanic Cloud, searching for microlensing events in this high-density stellar source environment, with a large cross section through the dark matter halo. Particularly relevant to this discussion, the combined analysis of the MACHO and EROS surveys demonstrated that $\lesssim 25 \%$ of the dark halo is made of planetary-mass MACHOs in the mass range between roughly 0.3 times the mass of Mars and the mass of Jupiter, the first such constraints on the abundance of planetary-mass objects in the halo of our Galaxy (Alcock et al. 1996). See Moniez (2010) for a comprehensive history of these efforts.

Once MACHOs were largely ruled out as a dark matter candidate, microlensing surveys began to focus on lines of sight toward the Galactic bulge to constrain Galactic structure (Paczyński 1991) and search for bound exoplanets (Mao \& Paczynski 1991; Gould \& Loeb 1992). Initially, these surveys lacked the field of view to both find relatively rare microlensing events and monitor them with sufficient cadence to detect the much shorter (and unpredictable) planetary perturbations. Instead, a two-tier system was employed, wherein the survey teams used relatively low cadence observations to alert followup observers of ongoing microlensing events. The relatively small numbers of ongoing microlensing events could then be monitored at much higher cadence by collaborations with access to a longitudinally distributed suite of telescopes. See Gaudi (2012) for a review of the history of microlensing surveys for exoplanets during this phase of the field.

Eventually, the MOA and OGLE surveys, along with the (more recently formed) Korea Microlensing Network (KMTNet; Kim et al. 2016) survey, have developed the capability to monitor the Galactic bulge with sufficient cadence to simultaneously detect isolated microlensing events and search for perturbations due to bound planets. This resulted in the first tentative detection of an excess of $\sim 1$-day-long events, which implied a substantial population of Jupiter-mass FFPs with an inferred abundance of roughly two free-floating Jupiter-mass planets per star in the Galaxy (Sumi et al. 2011).

This result was later challenged by Mróz et al. (2017), who placed an upper limit of $\lesssim 0.25$ Jupiter-mass FFPs per star. Notably, though, Mróz et al. (2017) did find tentative evidence of an excess of very short timescale events $\left(t_{\mathrm{E}} \lesssim 0.5\right.$ days), possibly indicating a population of free-floating or wideseparation Earth-mass planets, although it is important to note that these events were generally poorly sampled and thus have large uncertainties in their timescales. They therefore may be spurious. Regardless, these efforts demonstrate the potential of Galactic bulge microlensing surveys to find free-floating or widely bound planetary-mass objects.

Indeed, quite recently, multiple well-characterized, extremely short microlensing events have been discovered. Mróz et al. (2018, 2019a), and Mróz et al. (2020) together report a total of four FFP candidates, two of which had timescales consistent with Earth- or Neptune-mass lenses. Han et al. (2020a) report the discovery of three events consistent with brown dwarf mass lenses (masses of $\sim 0.04 M_{\odot}$ ), of which two are isolated and one is in a near-equal-mass binary. An important caveat for candidate FFP events is the potential to exclude any potential host stars. If the separation of a planet and its host is sufficiently large ( $\gtrsim 10 \mathrm{au}$; Han et al. 2005) and the geometry is correct, the source can appear to be magnified by an effectively isolated planet. Thus, wide-separation planets can masquerade as FFPs in a subset of microlensing events. 
This has been discussed before by several authors (Di Stefano \& Scalzo 1999; Han \& Kang 2003; Han et al. 2005), all of which propose pathways to determine whether a planetary-mass lens is bound or free-floating. Mróz et al. (2018, 2019a) place limits on the presence of a host photometrically, but detailed modeling of the magnification curve and photometric follow-up can also be used to determine whether the lens is isolated (Han \& Kang 2003; Han et al. 2005; Henderson \& Shvartzvald 2016). As an example, detailed modeling has been used to determine the true, bound nature of FFP candidates by Bennett et al. (2012) and Han et al. (2020b).

Keeping in mind these caveats, it has been demonstrated previously (Bennett \& Rhie 2002; Strigari et al. 2012; Penny et al. 2013, 2017; Ban et al. 2016; Henderson \& Shvartzvald 2016) that a space-based microlensing survey will have unprecedented sensitivity to short-timescale microlensing events owing to FFP lenses that have masses comparable to our Moon or greater. We investigate this opportunity more fully here, as applied to NASA's next flagship mission, the Nancy Grace Roman Space Telescope (Roman).

\subsection{The Nancy Grace Roman Space Telescope and Its Galactic Exoplanet Survey}

Initially called the Wide Field Infrared Survey Telescope (WFIRST; Spergel et al. 2015), Roman is currently planned to conduct three Core Community Surveys: the High Latitude Survey (Troxel et al. 2019), the Type Ia Supernova Survey (photometric, Hounsell et al. 2018; and spectroscopic), and the Galactic Exoplanet Survey (Penny et al. 2019). These surveys will be accompanied by a Guest Observer program (including notionally $25 \%$ of observing time) and a demonstration of numerous new-to-space technologies with the Coronagraph Instrument (CGI; Debes et al. 2016; Bailey et al. 2019).

The surveys currently have notional designs that will allow them to make key measurements that will in turn provide unique constraints on the nature and time evolution of dark matter and dark energy, as well as provide novel constraints on the demographics of cold exoplanets (Akeson et al. 2019). The designs of these surveys are notional in that the final observing program will not be settled on until much closer to launch and, importantly, will incorporate community input.

For the Roman Galactic Exoplanet Survey, Roman will use the microlensing technique to search for bound planets with mass roughly greater than that of Earth $\left(M_{\oplus}\right)$ with semimajor axes in the range of $\sim 1-10$ astronomical units $(\mathrm{au})^{7}{ }^{7}$ At planethost star separations roughly equivalent to the Einstein radius of the lens system (and thus peak sensitivity), Roman will be able to detect planets with masses as low as roughly twice the mass of the Moon, roughly the mass of Ganymede (Penny et al.

2019, hereafter Paper I). Through finding these planets near and beyond the water snowline of host stars, Roman will complement the parameter space surveyed by Kepler (Borucki et al. 2010). When combined, these broad, monolithic surveys promise to provide the most comprehensive view of exoplanet demographics to date and thus provide the fundamental empirical data set by which predictions of planet formation theories can be tested (Penny et al. 2019).

\footnotetext{
7 Roman will also discover $\gtrsim 100,000$ planets with periods $\lesssim 64$ days using the transit technique (Montet et al. 2017).
}

The current version of the Roman microlensing survey area covers approximately $2 \mathrm{deg}^{2}$ near the Galactic bulge, composed of seven fields covered by the $0.282 \mathrm{deg}^{2}$ field of view of the Wide Field Instrument (WFI; Spergel et al. 2015). Throughout the survey, it will observe $\sim 50,000$ microlensing events, of which roughly 1400 are predicted to show planetary perturbations (Paper I). The current notional survey design includes six 72-day seasons, clustered near the beginning and end of the $5 \mathrm{yr}$ primary lifetime of the mission. Each season will be centered on either the vernal or autumnal equinoxes, when the Galactic bulge is visible by Roman.

During a season, Roman will perform continual observations using its wide 1-2 $\mu \mathrm{m}$ W146 filter at 15-minute cadence. Each visit will have a $46.8 \mathrm{~s}$ W146 exposure of the WFI that will reach a precision of 0.01 mag at $W 146 \approx 21$. These observations will be supplemented with at least one and likely two narrower filters (yet to be decided), which will sample the fields at much lower cadence. Paper I assumed observations with only one additional (Z087) filter with a $12 \mathrm{hr}$ cadence, but this observing sequence has not yet been finalized. When a microlensing source star is sufficiently magnified and observations are taken in more than one filter, Roman will be able to measure the color of the microlensed source star. Measurement of the source color and magnitude can be used to constrain the angular radius of the source star $\theta_{*}$, which can be be used to measure $\theta_{\mathrm{E}}$ if the event exhibits finite-source effects (Yoo et al. 2004). For more details on the currently planned Roman hardware, the microlensing survey design, and the bound planet yield, the reader is encouraged to read Paper I.

\subsection{Constraining the Abundance of Free-floating Planets with Roman}

The properties of Roman and the Galactic Exoplanet Survey design that make it superb at detecting and characterizing bound planets are the same properties that allow it to detect and characterize FFPs. FFPs can produce events lasting from $\sim 1 \mathrm{hr}$ to $\sim 1$ day. Many of the same observables for bound planet microlensing events are also desirable for FFPs, such as the source color and brightness, which can constrain the angular source size and the mass of the lensing body. Measuring the mass of an isolated lens requires additional measurements of event parameters (Gould \& Welch 1996) and would require supplementary and simultaneous ground-based or space-based observations (e.g., by EUCLID, Zhu \& Gould 2016; Bachelet \& Penny 2019; Ban 2020). We do not address parameter recovery through modeling or mass estimation of detected lenses, both of which are beyond the scope of this work.

The goal of this work is to predict Roman's ability to measure the distribution of short-timescale events attributed to free-floating planets. To do so, we will briefly revisit the microlensing survey simulations presented in Paper I and detail the changes we made to them in Section 2. We then examine light curves Roman will detect in Section 3. Section 4 will contain a discussion of the yield and limits Roman will place on FFPs in the Milky Way (MW). Finally, we will discuss our findings and conclude in Sections 5 and 6. We include two appendices, one that provides a primer on the phenomenology of microlensing events in the regime where the angular size of the source is much greater than the angular Einstein ring radius (Appendix A), and a second exploring the sensitivity of Roman's yield to the detection criteria we impose (Appendix B). 
Table 1

Roman Galactic Exoplanet Survey Parameters

\begin{tabular}{lc}
\hline \hline Parameter & Value \\
\hline Area & $1.97 \mathrm{deg}^{2}$ \\
Baseline & $4.5 \mathrm{yr}$ \\
Seasons & $6 \times 72 \mathrm{days}$ \\
Fields & 7 \\
Avg. slew and settle & $83.1 \mathrm{~s}$ \\
Primary (W146) filter & $0.93-2.00 \mu \mathrm{m}$ \\
Exposure time & $46.8 \mathrm{~s}$ \\
Cadence & $15 \mathrm{minutes}$ \\
Total exposures & $\sim 41,000 \mathrm{per}$ field \\
Secondary (Z087) filter & $0.76-0.98 \mu \mathrm{m}$ \\
Exposure time & $286 \mathrm{~s}$ \\
Cadence & $\lesssim 12 \mathrm{hr}$ \\
Total exposures & $\sim 860 \mathrm{per}$ field \\
Phot. precision & $0.01 \mathrm{mag} @ W 146 \sim 21.15$ \\
\hline
\end{tabular}

Note. A summary of the Cycle 7 design is fully detailed in Paper I. This is the current design and is subject to change prior to the mission. For example, the exposure time and cadence of observations in the Z087 and other filters have not been set; we have assumed a $12 \mathrm{hr}$ cadence here, but observations in the other filters are likely to be more frequent.

\section{Simulations}

To simulate the Roman microlensing survey, we use the free-floating planet module of the GULLS microlensing simulator (Penny et al. 2013, 2019). Here we only briefly discuss how FFP simulations differ from the bound planet simulations of Paper I. We use the mission and survey parameters for the Cycle 7 design as fully detailed in Paper I and summarized in Table 1.

GULLS simulates individual microlensing events by combining pairs of source and lens stars drawn from a population synthesis Galactic model (GM). We use the same GM as Penny et al. (2013) and Paper I, version 1106 of the Besançon model, for consistency between our results. Version 1106 is intermediate between the two publicly available Besançon models (Robin et al. 2003, 2012) and is described fully in Penny et al. (2013) and Paper I. The usefulness of population synthesis GMs for microlensing was first demonstrated by Kerins et al. (2009). An updated model by Specht et al. (2020) has recently been shown to provide a high level of agreement with the 8000-event OGLE-IV event sample of Mróz et al. (2019b).

GULLS simulates Roman's photometric measurements by injecting GM stars, including the source, into a synthetic postage stamp image. From this image the photometric precision as a function of magnification is computed assuming a $3 \times 3$ pixel square aperture centered on the microlensing event.

The actual Roman photometric pipeline will be much more sophisticated than this, using both point-spread function (PSF) fitting and difference image analysis to perform photometry. Aperture photometry is likely somewhat conservative relative to PSF fitting photometry in terms of photon noise, but this is offset by optimism in not dealing with relative pixel phase offsets with an undersampled PSF (see Paper I for a full discussion). The model microlensing light curve is computed from a finite-source point-lens model (Witt \& Mao 1994) with no limb darkening. The realistic, color-dependent redistribution of surface brightness from limb darkening will modify the light-curve shape of events in which finite-source effects are present (Witt 1995; Heyrovský 2003) but does not significantly affect detection probability. We briefly discuss the impact of omitting limb darkening from our simulations in Section 5.5.

Our simulations follow those of Paper I almost exactly, but we replace the stellar lenses drawn from the catalogs generated from the GM with an isolated planetary-mass object and assume zero flux from the injected lens. This results in all simulated events having planetary-mass point lenses with the velocity and distance distributions of stars in the GM. One might expect small differences in the phase-space distributions between stars and FFPs, depending on their origin, but we do not account for this in this study (e.g., van Elteren et al. 2019 found that FFPs are ejected from clusters with larger velocities than escaping stars, but only by a few kilometers per second, which is much less than typical $\sim 100 \mathrm{~km} \mathrm{~s}^{-1}$ relative velocities between lens and source).

The source and lens of each simulated microlensing event are drawn from GM catalogs that represent a $0.25 \times 0.25 \mathrm{deg}^{2}$ area of sky, which we call a sight line. Each event $i$ is assigned a weight $w_{i}$ proportional to the event's contribution to the total event rate along a sight line,

$$
w_{i}=0.25^{2} \operatorname{deg}^{2} f_{1106, \operatorname{Roman}} \Gamma_{\operatorname{deg}^{2}} T_{\operatorname{sim}} u_{0, \max , i} \frac{2 \mu_{\mathrm{rel}, i} \theta_{\mathrm{E}, i}}{W},
$$

where $T_{\text {sim }}=6 \times 72$ days is the total Roman microlensing survey duration, $u_{0, \max , i}$ is the maximum impact parameter for each simulated event, $\Gamma_{\mathrm{deg}^{2}}$ is the sight line's microlensing event rate per square degree, $f_{1106 \text {, Roman }}$ is a correction factor, and $W$ is a normalization factor defined below. The $\Gamma_{\mathrm{deg}^{2}}$ event rates were calculated by Monte Carlo integration using catalogs of source and lens stars drawn from the GM.

We use the same $f_{1106, \text { Roman }}=2.81$ as in Paper I, which matches the GM's event rate to the microlensing event rate measured using red-clump source stars by Sumi et al. (2013) and corrected by Sumi \& Penny (2016). Mróz et al. (2019b) measured microlensing event rates with a larger sample of events from the OGLE-IV survey. They measured the event rate per star for source stars brighter than $I<21$ (a so-called all-star event rate), which was consistent with the Sumi \& Penny (2016) red-clump event rate, but which was a factor of 1.4 smaller than MOA's all-star event rate estimated by Sumi \& Penny (2016) with sources brighter than $I<20$. We elect to maintain the same event rate scaling as Paper I because the origin of the discrepancy in all-star rates between Mróz et al. (2019b) and Sumi \& Penny (2016) is not clear, and for reasons discussed in Paper I we expect that the small bar angle in the GM may cause an overcorrection if corrections are tied to allstar event rates.

For each event, $u_{0}$ is uniformly drawn from $\left[0, u_{0, \max }\right]$, where $u_{0, \max , i}=\max (1,2 \rho)$ and $\rho=\theta_{*} / \theta_{\mathrm{E}}$ is the angular radius of the source star relative to $\theta_{\mathrm{E}}$. We impose the $2 \rho$ alternative to ensure that all lens transiting source events are simulated. We also ran supplemental simulations at higher masses with $u_{0, \max , i}=\max (3,2 \rho)$ and found consistent event rates with those used $u_{0, \max , i}=\max (1,2 \rho)$. This event weight should be normalized to the stellar-lens event rate, so we divide $\theta_{\mathrm{E}, i}$ by the mass ratio of the injected lens $\left(M_{p, i}\right)$ and the star that it is replacing $\left(M_{*, i}\right), \sqrt{q_{i}}=\sqrt{M_{p, i} / M_{*, i}}$, to correct the value. We note that this methodology is equivalent to the assumption that there is one FFP per star in the Galaxy. 
The normalization factor is then defined as

$$
W=\sum_{i} \frac{2 \mu_{\mathrm{rel}, i} \theta_{\mathrm{E}, i}}{\sqrt{q_{i}}},
$$

such that the sum of all simulated event weights would have equaled the number of events occurring over the survey duration had each stellar lens not been replaced by an FFP.

We run two sets of simulations, both of which have lens masses drawn from $\log \left(M / M_{\oplus}\right) \in[-5,5]$ (i.e., $0.5 \%$ the mass of Pluto to $0.3 M_{\odot}$ ). In the first set of simulations, we simulate equal numbers of planets with a range of discrete masses uniformly spaced by $0.25 \mathrm{dex}$. In the second set, we draw log-uniform random-mass lenses from the same range. In both cases we draw events until the error on the estimated event rate due to random sampling and accounting for unequal event rates is less than $0.1 \%$.

\subsection{Detection Criteria}

We use two detection criteria for microlensing events. The first is the difference in $\chi^{2}$ of the observed light curve relative to a flat (unvarying) light-curve fit

$$
\Delta \chi^{2}=\chi_{\text {Line }}^{2}-\chi_{\mathrm{FSPL}}^{2}
$$

where $\chi_{\text {Line }}^{2}$ is the $\chi^{2}$ value of the simulated light-curve data for a flat line at the baseline flux and $\chi_{\mathrm{FSPL}}^{2}$ is the same but for the simulated data to the true finite-source point-lens model of the event.

The second criterion is that $n_{3 \sigma}$, the number of consecutive data points measured at least $3 \sigma$ above the baseline flux, must be greater than 6 , i.e.,

$$
n_{3 \sigma} \geqslant 6 .
$$

This criterion serves two purposes. First, it mimics the type of selection cut that previous free-floating planet searches have used to minimize the number of false positives caused by multiple consecutive outliers from long-tailed uncertainty distributions (e.g., Sumi et al. 2011; Mróz et al. 2017). Second, it ensures that any events detected will stand a good chance of being modeled with four or five free parameters without overfitting. Extremely short events and those with large $\rho$ may suffer from degeneracies where even six data points may be insufficient to correctly model the event (S. A. Johnson et al. 2020, in preparation). Naively, the probability of six consecutive data points (assuming that they are Gaussian distributed) randomly passing this criterion is $\sim(1-0.9987)^{6} \approx 10^{-18}$. Given the number of data points per light curve $\left(\sim 4 \times 10^{5}\right)$ and the number of light curves $\left(\sim 2 \times 10^{8}\right)$, we expect a by-chance run of six points more than $3 \sigma$ to occur at a rate of $\sim 4 \times 10^{-4}$ per survey. Thus, this may appear to be an overly conservative detection criterion. However, it is likely that neighboring data points will not be strictly uncorrelated, and therefore it is important to adopt conservative detection criteria. For example, a falsepositive event may be caused by hot pixels, which are obviously correlated with time. We briefly discuss the possibility of contamination by this and other false positives in Section 5.2. We further motivate these selection criteria in the next section.
Our predictions for the yields of detectable free-floating planets are calculated using the weights defined in Equation (2) modified by a Heaviside step function for each detection criterion,

$$
N_{\mathrm{det}}=\sum_{i} w_{i} H\left(\Delta \chi^{2}-300\right) H\left(n_{3 \sigma}-6\right) .
$$

\section{Light Curves of Free-floating Planets as Seen by Roman}

The continual coverage provided by Roman enables the detection of the microlensing events caused by free-floating planets without the difficulties faced by ground-based microlensing surveys. In this section we explore the light curves of free-floating planets that Roman might detect, covering a wide range of planet masses.

We begin with large-mass FFPs and brown dwarfs, which can be challenging to observe from the ground owing to their event timescales being comparable to several days. Figure 1 shows the light curve for a brown dwarf mass lens in the top panel. This example has a relatively long timescale compared to what is expected for typical free-floating planet events, but we include it as an extreme case to demonstrate the confusion with stellar-lens events. These cases display the density of Roman photometry, especially in the bottom panel, which has nearly $10003 \sigma$-significant W146 measurements in the time span of roughly 6 days. These events will be extremely well characterized and are nearly guaranteed to have color measurements while the source is magnified.

Figure 2 show the light curves of events at the opposite end of the detectable FFP mass spectrum. A very low mass lens exhibits modest finite-source effects in the top panel. Much stronger finite-source effects are apparent in the bottom panel for a giant source with $\rho \approx 10$. In the latter case, the magnification saturates at the expected value of $1+2 / \rho^{2}$ (Equation (A2)), i.e., just 1.02 in the absence of limb darkening. To demonstrate the impact of limb darkening for this event, we use the same event parameters to recompute the magnification using the Lee et al. (2009) method as implemented in MulensModel (Poleski \& Yee 2018). This is shown as the gray long-dashed line underlying the simulated event. The peak is higher than in the event without limb darkening, and the "shoulders" of the top hat drop modestly. Even for such extreme finite-source events the impact of limb darkening will be modest on the number of events that pass selection cuts. Both events highlight the precision of Roman photometry. The light curves in Figures 1 and 2 are chosen to demonstrate a number of morphologies, photometric precisions, masses, and detection significances of Roman events.

For a broader, more representative look at the events Roman will detect, Figure 3 displays an ensemble of light curves for each of the five discrete mass lenses we consider. In each panel, we randomly select 100 events that passed our detection criteria in $\Delta \chi^{2}$ and $n_{3 \sigma}$. We then normalize the transparency of each curve to the maximum weight of those events included (Equation (2)). In this way, darker curves indicate events that contribute more to the calculated event rate. We place vertical dashed lines at the positive/negative weighted average of $t_{\mathrm{E}}$ for these subsets, as well as a horizontal row of gray dashes below the curves representing the W146 cadence (15 minutes) in the three rightmost panels. Note that the scales of the horizontal axes shrink with decreasing mass (as $t_{\mathrm{E}} \propto M^{1 / 2}$ ), but we maintain the scale between the two rightmost panels. At higher 

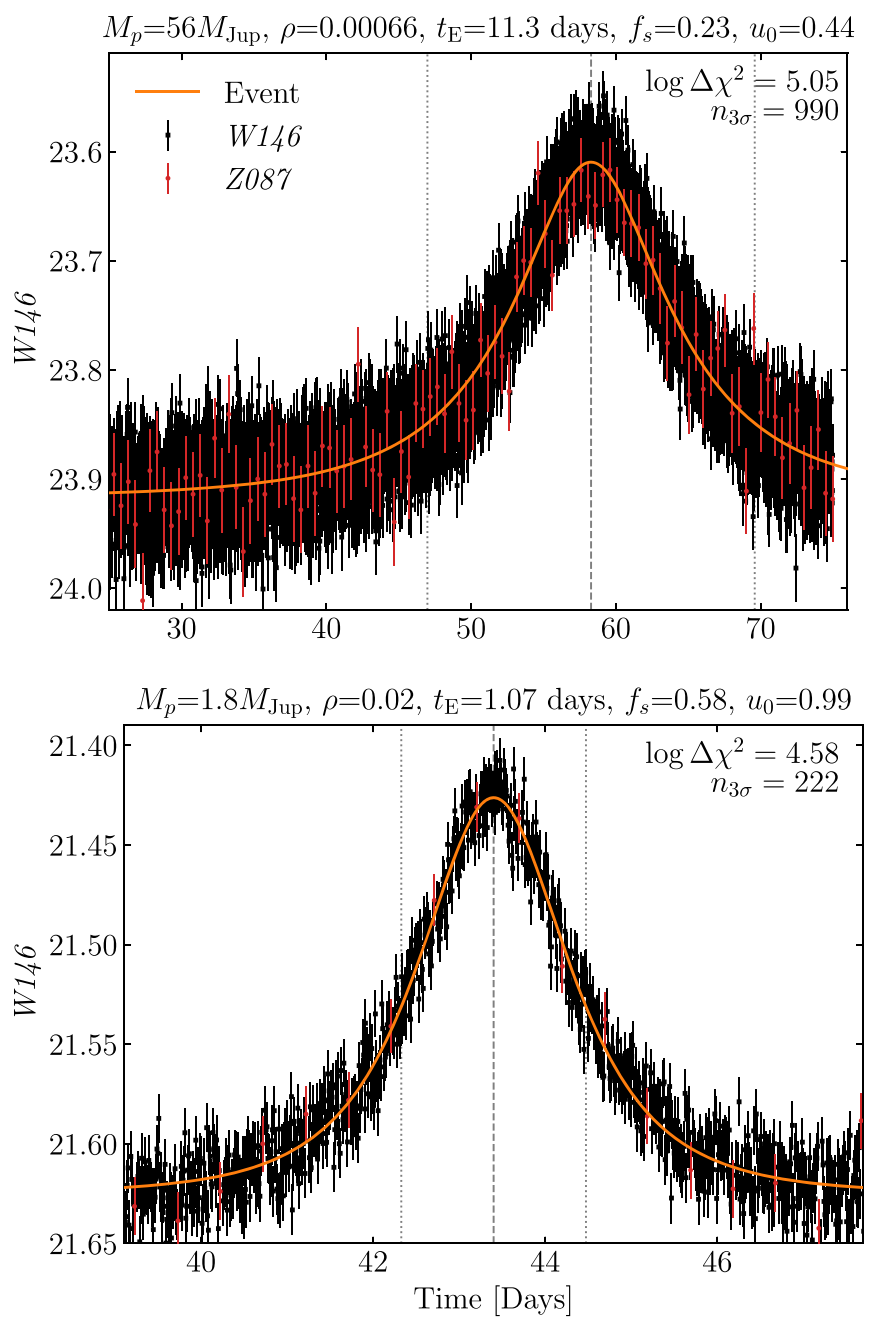

Figure 1. Two examples of simulated events as observed by Roman. Black (red) points are observations in the W146 (Z087) filters, and the overlying orange line is the input lensing model. Above each panel, $M_{p}$ is the mass of the lens in Jupiter masses $\left(M_{\mathrm{Jup}}\right)$ or Earth masses $\left(M_{\oplus}\right), \rho$ is the angular size of the source normalized to the Einstein ring, $t_{\mathrm{E}}$ is the Einstein timescale of the event, $f_{s}$ is the blending parameter, and $u_{0}=\theta_{0} / \theta_{\mathrm{E}}$ is the minimum impact parameter. We also include the values of $\log \Delta \chi^{2}$ and $n_{3 \sigma}$ light curve. Vertical shortdashed gray lines indicate $\pm t_{\mathrm{E}}$ values of the event, and the long-dashed gray line indicates the peak of the event. The expected photometric precision and 15 -minute cadence for observations in the primary W146 band will make detection of such events trivial. Top: an event with a $\sim 60 M_{\text {Jup }}$ brown dwarf lens. Bottom: an event with a $\sim 2 M_{\text {Jup }}$ mass lens.

masses $\left(\geqslant 10^{2} M_{\oplus}\right)$ the light curves look like one would expect for point-like sources. As the mass of the lens decreases, a larger fraction of detected events exhibit finite-source effects as described in Appendix A.

Figure 4 shows the rightmost panel of Figure 3 with both axes rescaled in order to show finer detail for the lowest-mass lenses. However, note that the magnification axis remains logarithmic. Note that there are only five dashes (five photometric measurements) during the expected duration $\left(2 t_{\mathrm{E}}\right)$, marked by the vertical gray dashed lines. However, the true duration of these events is often considerably longer. Were there no finite-source effects, the events of low-mass lenses would often be too short to accurately model with the 15minute cadence of the W146 band, but because the sourcecrossing time for these sources can be a factor of several times longer than $2 t_{\mathrm{E}}$, these events may be well characterized.


Figure 2. Same as Figure 1, but for two very low mass lenses. We note that, although both events contain one measurement taken in the Z087 filter, this is not representative of most low-mass lens events. Top: illustrative light curve due to a roughly Mars-mass FFP, with relatively mild finite-source effects. Bottom: illustrative light curve due to a $\sim 0.6 M_{\oplus}$ FFP, in this case lensing a giant source, thereby exhibiting strong finite-source effects. Note that, in this case, the fact that the source is a giant results in nearly no blending and the large value of $\rho$. In such cases, the magnification would saturate at $1+\frac{2}{\rho^{2}}$ (shown as the orange line) in the absence of limb darkening. However, when we include limb darkening, the light curve would appear as a long-dashed gray line (for $\Gamma=0.4$ ).

\subsection{Detection Thresholds}

Given the potential challenges involved in detecting short events, we revisit the detection criteria we presented in Section 2 to ensure that they fulfill their purpose. We require that $\Delta \chi^{2}$ of an event be at least 300 and that the event have an $n_{3 \sigma}$ of at least 6 . These thresholds are similar in nature to the initial cuts placed by Sumi et al. (2011) and Mróz et al. (2017). Both use $n_{3 \sigma} \geqslant 3$ and a statistic $\chi_{3+}=\sum_{i}\left(F_{i}-F_{\text {base }}\right) / \sigma_{i}$ to quantify the significance of candidate events, where $F_{i}$ is the $i$ th data point within an event with uncertainty $\sigma_{i}$ and $F_{\text {base }}$ is the baseline flux. Sumi et al. (2011) use $\chi_{3+} \geqslant 80$, while Mróz et al. (2017) relaxed this to $\chi_{3+} \geqslant 32$ owing to the typically higher quality of the OGLE data.

It is not straightforward to compare our cuts to the $\chi_{3+}$ criteria of Sumi et al. (2011) and Mróz et al. (2017); however, we can consider an extreme case. Imagine an event that barely 


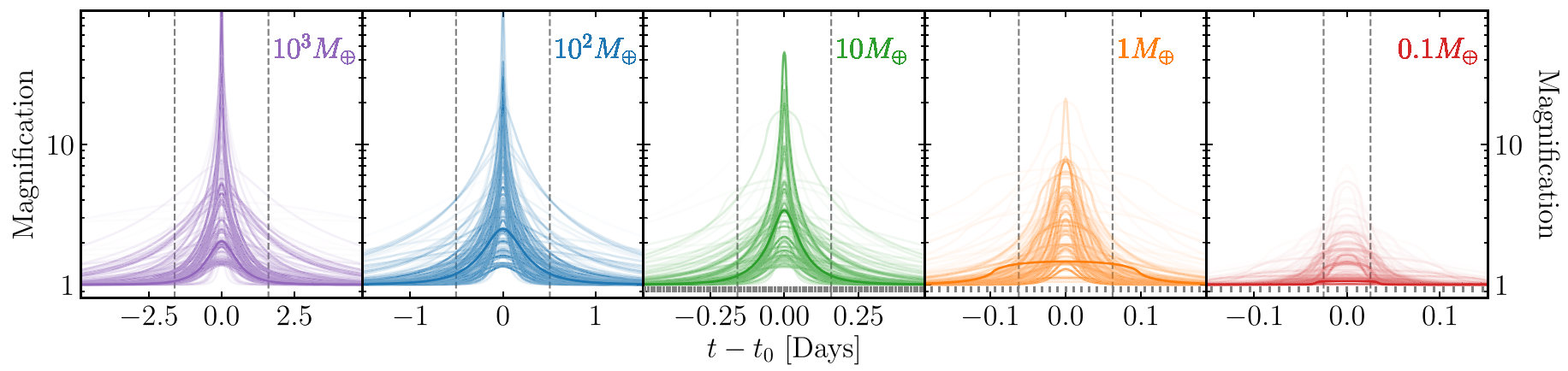

Figure 3. Samples of simulated magnification curves from events detectable by Roman at each mass of $10^{3}, 10^{2}, 10,1$, and $0.1 M_{\oplus}$, from left to right. For each mass, we randomly select 100 events that passed our detection criteria and plot their magnification curves. The weighted average $\pm t_{\mathrm{E}}$ is indicated by the vertical dashed lines in each panel. Note that the horizontal axis scale changes as mass decreases and the vertical axis uses a logarithmic scale. The black, horizontal tick marks below the curves indicate the $W 146$ cadence; we note that they are only shown for masses of $10 M_{\oplus}$ and below. The transparency of each curve is proportional to the weight of the event normalized to the maximum weight of events included in the panel. In this way, darker lines exemplify events that will contribute more to the event rate for that mass bin.

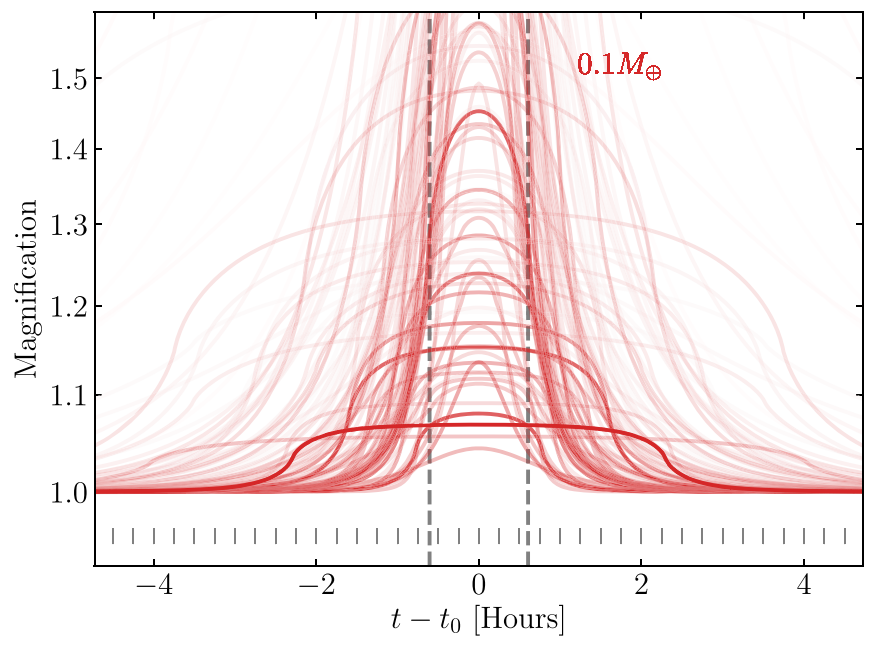

Figure 4. Same as the rightmost panel of Figure 3, but rescaled to highlight the finer detail of light curves arising from $\sim 0.1 M_{\oplus}$ lenses. Note that the magnification remains in log-scale, but the horizontal axis has been converted from days to hours. The vertical dashed lines are the weighted average $t_{\mathrm{E}}$, which indicate that the Einstein timescales are generally much shorter than the observed timescales, which are set by the crossing time of the source when $\rho \gg 1$. The gray vertical dashes match the 15 -minute observing cadence of the W146 band.

passes both our criteria with $\Delta \chi^{2}=300$ and $n_{3 \sigma}=6$, but with a minimal $\chi_{3+}$. This event would have six consecutive data points, five at $3 \sigma$ and a single data point at $16 \sigma$, making a total $\Delta \chi^{2}=301$. This particular event would then have a value of $\chi_{3+}=31$, barely failing to pass the Mróz et al. (2017) $\chi_{3+}$ threshold. More realistic events would have higher values of $\chi_{3+}$; therefore, our cuts are at least comparable to those used in Mróz et al. (2017) but are likely slightly more stringent. We also expect fewer systematics and less correlated noise in Roman data compared to those of ground-based surveys.

Sumi et al. (2011) and Mróz et al. (2017) follow their initial cuts with several more to further vet their samples, ensuring that each is truly a microlensing event. These include (among others) the rejection of light curves with more than a brightening event, the rejection of light curves with poor goodness-of-fit statistics to initial models, and the rejection of events that did not have the rise or fall of the event sufficiently sampled. Without a detailed investigation of the uncertainties in

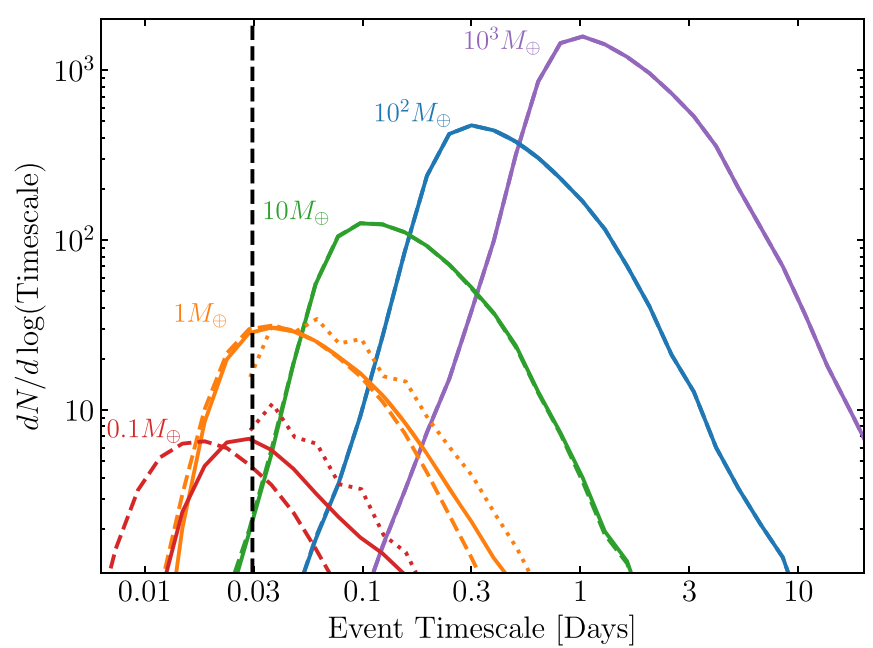

Figure 5. Distribution of detected events as a function of timescale for different lens mass populations. We plot the distributions as a function of $t_{\mathrm{E}}$ as dashed lines. The solid lines are the distributions as a function of the maximum of $t_{\mathrm{E}}$ or the source half-chord-crossing time, $0.5 t_{c}$. These distributions are nearly identical for masses above $10 M_{\oplus}$ because for these masses typically $t_{\mathrm{E}} \gg 0.5 t_{c}$, whereas for lower masses the timescale is largely set by the source chord-crossing time. For the two lowest masses, we also plot as dotted lines the distribution of $\frac{1}{2} n_{3 \sigma} \times 15$ minutes. The vertical dashed line indicates $3 \times$ the W146 band cadence. The cut we impose on $n_{3 \sigma}$ (e.g., the dashed vertical line) eliminates events that are formally "significant" according to the $\Delta \chi^{2}$ criterion but would likely be poorly characterized owing to the small number of significant points. Interestingly, as a result of the fact that the effective event timescale saturates at the source chord-crossing time for low-mass lenses, many events pass our cuts that would not in the absence of finite-source effects.

the observables, we must use heuristic cuts to approximate these detailed investigations. We have not implemented these further cuts because our simulations do not contain the false positives they are designed to reject. We do explore the thresholds we place in Appendix B, and we determine scaling relations to predict how loosening or tightening these thresholds will impact Roman's free-floating planet yield. These relations can also be used to estimate the change in yield as the microlensing survey design evolves.

We examine how our thresholds of $\Delta \chi^{2} \geqslant 300$ and $n_{3 \sigma} \geqslant 6$ impact the timescale distribution of events in Figure 5, where we assume delta functions in mass (one planet per star) for each mass shown. First, we plot the distribution of events as a 


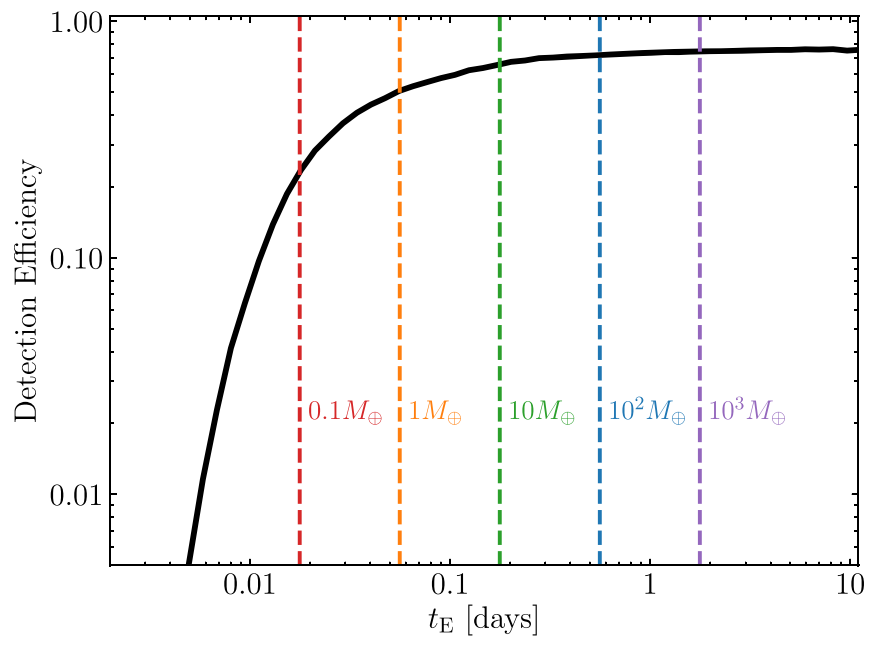

Figure 6. Roman's detection efficiency as a function of timescale (black solid line) computed as the fraction of the events that pass our detection criteria relative to all events. Roman will have $>50 \%$ detection efficiency down to events with timescales as short as $1.5 \mathrm{hr}$. The five vertical lines indicate typical timescales for lenses with the mass indicated.

function of $t=\max \left(t_{\mathrm{E}}, \frac{1}{2} t_{c}\right)$ as solid lines. Here $t_{c}$ is the source chord-crossing time as defined in Equation (A3). ${ }^{8}$

These distributions are meant to show the duration of events detected. Events that exhibit extreme finite-source effects (and thus have "top-hat" light curves) $t_{\mathrm{E}}$ will be less than $\frac{1}{2} t_{c}$, and the event will be longer than expected. This will allow for the detection of events that would not be typically detectable were there no finite-source effects.

Second, we plot the distribution as a function of solely the $t_{\mathrm{E}}$ values of events as dashed lines. There is essentially no difference between these distributions for lens masses $\geqslant 10 M_{\oplus}$, but we see a strong offset between the solid- and dashed-line distributions for the $0.1 M_{\oplus}$ events. For low-mass lenses, this demonstrates the previous point that some detected events would have expected timescales much shorter than would be detectable considering our requirement on $n_{3 \sigma}$.

Finally, for the two lowest masses we show the distribution as a function of $t_{3 \sigma}=\frac{1}{2} n_{3 \sigma} \times 15$ minutes, half the length of the event while significantly magnified. These distributions have no events less than 45 minutes (the vertical black dashed line), which is indicative of our detection criteria on $n_{3 \sigma}$. For the $0.1 M_{\oplus}$ events, the event timescale saturates at the source chord-crossing timescale for many events, pushing the distribution toward longer durations. This is even more enhanced when considering the distribution while significantly magnified.

More broadly, we show the detection efficiency as a function of the microlensing timescale $t_{\mathrm{E}}$ in Figure 6. The black line is the number of detected events relative to the number of injected events with a given timescale within a single 72-day season. The typical timescales for lenses of five different masses are illustrated with vertical lines. Within a season, Roman will maintain a $\gtrsim 50 \%$ efficiency down to $t_{\mathrm{E}} \approx 1.5 \mathrm{hr}$. This efficiency would be proportionately lower if we consider the efficiency over the entire $5 \mathrm{yr}$ baseline by a factor of

\footnotetext{
8 Typically the source radius crossing time as defined by $t_{*}=\rho t_{\mathrm{E}}=\theta_{*} / \mu_{\text {rel }}$ is used as a proxy for the timescale of the event (e.g., Skowron et al. 2011); however, we account for nonzero impact parameter $u_{0, *}$ similar to Mróz et al. (2019a). We follow their definition, except we use the variable $t_{c}$ instead for their Equation (A1). See Appendix A.
}

$(6 \times 72$ days $) /(5 \times 365$ days $)=0.23$ if $t_{0}$ is uniformly distributed, since the Galactic bulge will only be observed for a fraction of a year.

Overall, in this section we have demonstrated that Roman will be able to detect a wide range and variety of shorttimescale microlensing events. This will impact the overall timescale distributions of microlensing events that Roman will detect, and it must be accounted for in determining the detection sensitivity used to infer the true underlying distribution of event timescales, regardless of the nature of the lenses.

In the next section, we will present our predictions for the yield of and limits on free-floating planets given the fiducial Cycle 7 survey design.

\section{Predicted Yields and Limits}

In this section, we present our predictions for the number of FFPs Roman will detect, as well as the limits on the total mass of FFPs that can be set by Roman. Recall that the yields are calculated from summing the weights of simulated events that pass our detection cuts using Equation (6). We maintain our detection criteria of $\Delta \chi^{2} \geqslant 300$ and $n_{3 \sigma} \geqslant 6$, but we discuss the impact of changing these in Appendix B.

\subsection{Yield}

We must assume a mass function for FFPs if we are to estimate the number of FFPs that Roman will find. We assume two forms of mass function, one log-uniform in mass,

$$
\frac{d N}{d \log M_{p}}=1 \mathrm{dex}^{-1},
$$

and another inspired by an inferred mass function of bound planets detected by microlensing (following Cassan et al. 2012). In the second case, we assume that for low-mass planets the mass function saturates at two planets per star below $5.2 M_{\oplus}$. This prevents the number from "blowing up" as the planet mass decreases. The functional form is then

$$
\frac{d N}{d \log M_{p}}= \begin{cases}\frac{0.24}{\operatorname{dex}}\left(\frac{M_{p}}{95 M_{\oplus}}\right)^{-0.73} & \frac{M_{p}}{M_{\oplus}} \geqslant 5.2 \\ 2 \operatorname{dex}^{-1} & \frac{M_{p}}{M_{\oplus}}<5.2 .\end{cases}
$$

Note that Paper I used a function with the same mass dependence as the fiducial mass function for bound planets. This fiducial function is also consistent with the upper limits on the abundance of bound and wide-orbit planets measured by Mróz et al. (2017).

This mass function is somewhat optimistic compared to that found by Suzuki et al. (2016), who found that the mass ratio function is shallower for objects with mass ratio less than $\sim 2 \times 10^{-4}$ than that found by Cassan et al. (2012). This mass ratio corresponds to the typical mass ratio for a Neptune-mass planet. Nevertheless, we adopt the Cassan et al. (2012) mass function for continuity with Paper I.

We report the expected number of detections as a function of mass in Table 2. The first column ("One-per-star") assumes that there is a delta function of FFPs at that mass such that there is an equal number of FFPs to stars in the MW. The "Loguniform" and "Fiducial' columns assume bins that are $0.5 \mathrm{dex}$ in width for the two mass functions defined above. We use the 
Table 2

Expected Free-floating Planet Yields

\begin{tabular}{lccc}
\hline \hline Mass & \multicolumn{3}{c}{ Mass Function } \\
\cline { 2 - 4 }$\left(M_{\oplus}\right)$ & One-per-star & Log-uniform & Fiducial \\
\hline 0.01 & 1.22 & 0.349 & 0.698 \\
0.1 & 17.9 & 5.13 & 10.3 \\
1 & 88.3 & 25.2 & 50.5 \\
10 & 349 & 83.0 & 103. \\
100 & 1250 & 298 & 68.9 \\
1000 & 4100 & 976 & 42.0 \\
10,000 & 13,300 & 3170 & 25.4 \\
\hline Total & 3750 & 897 & 249 \\
\hline
\end{tabular}

Note. The "Total" row is an integration using the trapezoidal rule from 0.1 to $1000 M_{\oplus}$. The first and last rows are included for reference.

trapezoidal rule to integrate the number of detections with masses from 0.1 to $1000 M_{\oplus}$ to estimate the total yield of FFPs. We include rows for FFPs with masses of 0.01 and $10^{4} M_{\oplus}$ for reference. Were the mass function simply log-uniform, nearly 1000 free-floating planets would be detected. In the case of the fiducial mass function, we predict that Roman will detect roughly 250 FFPs.

Next, we consider how these populations will manifest in the timescale distribution of microlensing events measured by Roman. To start, we show the expected timescale distribution of detected stellar events with the same detection criteria $\left(\Delta \chi^{2} \geqslant 300, n_{3 \sigma} \geqslant 6\right)$ in Figure 7 . Note that the minimum mass included in the GM is $0.08 M_{\odot} \approx 80 M_{\text {Jup }}$ in the Galactic disk and $0.15 M_{\odot}$ in the Galactic bulge. Then, we consider three cases for populations of FFPs. The blue hatched region has an upper boundary that reflects the limit of at most 0.25 Jovian planets per star from Mróz et al. (2017). We also include the population of $5 M_{\oplus}$ free-floating or wide-separation planets that Mróz et al. (2017) cautiously consider as a possible explanation of the excess of very short timescale events. The orange shaded region has a lower (upper) bound corresponding to 5 (10) FFPs per star in the MW that are $5 M_{\oplus}$. Third, we show the expected distribution of detections using the continuous fiducial mass function in red. We also draw a realization of this mass function, which is included as the gray histogram with Poisson error bars.

If our fiducial assumptions are reasonable, Roman will be able to detect the signature of terrestrial-mass to Jovian-mass lenses in the event timescale distribution. With the lowest-mass planets giving rise to events with extended timescales owing to finite-source effects, the sensitivity is pushed to events with lens masses as low as a few times that of Mars. The fiducial mass function we use produces events detectable by Roman with timescales stretching over three orders of magnitude. These will leak into the timescale distribution attributable to the stars in the Galaxy, but because the model truncates at 0.08 $M_{\odot}$, there is no smooth transition.

\subsection{Limits}

If Roman detects no free-floating planets in a given mass range, it can still place interesting constraints on the occurrence rate of such planets, which in turn can be used to constrain planet formation theories. We can place expected upper limits on populations of FFPs using Poisson statistics, following Griest (1991). If we return to our delta function mass distribution such that we assume that there is one planet of that mass per star, we can place a 95\% confidence level upper limit for any mass bin, which corresponds to the situation in which we would expect fewer than three planets per star. Figure 8 plots the $95 \%$ confidence level Roman will be able to place on the total mass of bodies per star in the MW composed of bodies of mass $M$ if no lenses of that mass are detected. Note that the vertical axis is equivalent to $M_{p} d N / d \log M_{p}$ in units of $M_{\oplus}$. For comparison, we plot our fiducial mass function (Equation (8)) and the mass distribution for solar system bodies. ${ }^{10}$ The latter is to give some intuition as to whether there was an equivalent of a solar system's mass function worth of unbound bodies per star in the MW, but we note that such a mass function is likely to be incomplete at low masses, and possibly also at higher masses (Trujillo \& Sheppard 2014; Batygin \& Brown 2016). In other words, for typical planetary formation scenarios, a higher number of low-mass objects are ejected than remain in our solar system, and in at least a subset of planetary systems, a higher number of higher-mass objects are ejected than remain in our solar system.

This origin of the shape of the total mass limit curve deserves some discussion. For FFP masses $M \gtrsim 1 M_{\oplus}$ the curve rises as $M^{1 / 2}$, which is somewhat counterintuitive, though it may be recognized by those familiar with dark matter microlensing surveys. The number of expected microlensing events Roman will detect is set by the microlensing event rate $\Gamma$, which scales as the square root of the object mass $\Gamma \propto M^{1 / 2}$, if there is a fixed number of objects. But the vertical axis of Figure 8 is the total mass of expected objects of mass $M_{p}$ per star $M_{\text {tot }}$, not the total number. So for fixed $M_{\text {tot }}$, the number of objects scales as the inverse of the object mass $M^{-1}$, and thus the microlensing event rate produced by a fixed total mass of object scales with the individual object mass as $M^{-1 / 2}$. The total number of detections therefore scales as $N_{\text {det }} \propto M_{\text {tot }} M_{p}^{-1 / 2}$. The survey limit is a contour of a constant number of expected detections, and thus the total mass of ejected objected scales as $M_{\text {tot }} \propto M^{1 / 2}$.

Below $M \sim 1 M_{\oplus}$, the finite size of a typical Roman source star becomes larger than the typical Einstein ring radius of the lens, and so the event rate per object becomes independent of object mass. But the event rate per total object mass scales as $M^{-1}$, and we would expect the limit curve to become more steeply positive and scale as $M^{-1}$. However, the transition to the finite-source-dominated regime begins to reduce the peak magnification of events, even if lengthening them, which eventually significantly reduces the probability of a microlensing event being detected. Between $\sim 0.01$ and $1 M_{\oplus}$, finitesource effects from events with $1<u_{0}<\rho$ increase the detectable event rate (and reduce the total mass limit) by up to a factor of two relative to events with only $u_{0}<1$. Below $M \lesssim 0.01 M_{\oplus}$ finite-source effects decrease the maximum magnification of microlensing events to the point where they start to become undetectable, the detection efficiency begins to fall far faster than the event rate increases, and the slope of the limit curve inverts and becomes sharply negative.

Viewed broadly, the total mass limit curve shows that Roman will be an extremely sensitive probe of the total mass budget of loosely bound and free-floating masses. At its most

\footnotetext{
9 More specifically, if one expects three planets and detects none, according to the Poisson distribution, one could rule out the hypothesis that there are three planets at a significance of $1-\exp (-3) \simeq 95 \%$.

10 ssd.jpl.nasa.gov
} 


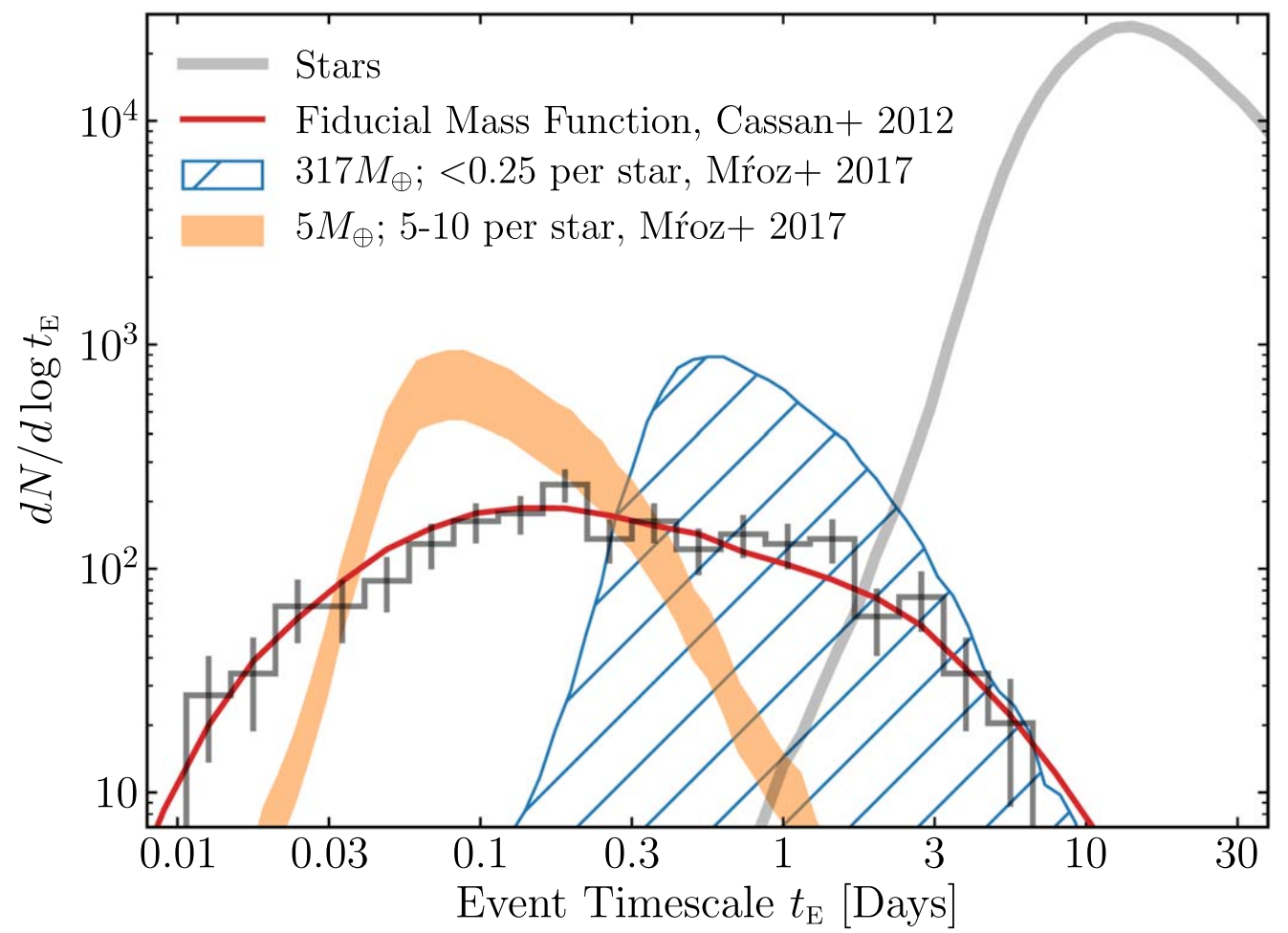

Figure 7. Roman will be able to measure the timescale distribution arising from FFP over three orders of magnitude in mass. The expected $t_{\mathrm{E}}$ signal from the stars is shown as a thick gray line. The blue hatched region marks the upper limit of 0.25 FFPs with mass $\sim M_{\text {Jup }}$ placed by Mróz et al. (2017). The orange region marks the expected number of events detected owing to $5 M_{\oplus}$ mass lenses assuming that there were 5 (lower bound) to 10 (upper bound) such FFPs per star in the Galaxy, as tentatively inferred by Mróz et al. (2017). The red line indicates the timescale distribution of detected FFPs using a fiducial mass function. The light-gray histogram with error bars is a realization of the simulated yield arising from that mass function, including Poisson uncertainties.

sensitive mass, $M \sim 3 \times 10^{-2} M_{\oplus}$ (near the mass of Mercury), Roman would be sensitive to total masses of just $\sim 0.1 M_{\oplus}$ per star (or roughly three objects per star). Roman will be sensitive to a total mass of $1 M_{\oplus}$ or less of objects with masses over a range of $\sim 0.003-100 M_{\oplus}$, or more than 5 orders of magnitude in mass. While for the lowest-mass objects these total masses are large compared to the mass budget of the present solar system, they are small compared to the total mass of planetesimal disks that are required to form solar-system-like planet configurations in simulations. For one example, the Nice model considers initial planetesimal disk masses between 30 and $50 M_{\oplus}$ beyond Neptune (Tsiganis et al. 2005). For a broader view of the expected population of loosely bound and free-floating objects, we can compare the Roman total mass limit curve to various predictions and constraints on these populations.

The first set of comparisons we draw is between Roman's limits and limits set by microlensing searches for MACHOs. There are three studies we consider:

1. As mentioned in the Introduction, Alcock et al. (1996) presented combined results of the MACHO and EROS microlensing surveys. These surveys were searching for MACHOs as candidates for the dark matter mass components of the MW halo.

2. Griest et al. (2014) found a similar limit on primordial black holes but used the Kepler transit survey. Kepler provides relatively high cadence observations of a fixed, relatively dense, stellar field, which is nearly optimal for a survey of microlensing events. The drawbacks were that this was toward a relatively low stellar density field compared to the LMC or the Galactic center and that potential sources were much closer than those typical of microlensing events. The limits placed here are from the analysis of $2 \mathrm{yr}$ of the Kepler mission, looking for shorttimescale events.

3. Niikura et al. (2019) used the Hyper Suprime-Cam on the Subaru Telescope (Subaru/HSC) to perform 2-minute cadence observations of M31 with high resolution. This search yielded the best constraint on low-mass primordial black holes as a component of the MW dark matter halo.

4. Niikura et al. (2019) placed limits roughly 50\% lower than the MACHO+EROS result at $50 M_{\oplus}$ using $5 \mathrm{yr}$ of OGLE-IV data. We do not include this result in Figure 8 as a result of space constraints.

These limits are not meant to be a direct comparison, so we simply scale their limits by assuming a stellar number density of $n_{\star}=0.14 \mathrm{pc}^{-3}$ and a dark matter halo mass density of $\rho_{\text {halo }}=0.3 \mathrm{GeV} / \mathrm{cm}^{-3}$. We determine their measured halo mass fractions, $f_{\mathrm{HM}}$, from their figures. Then, the mass of freefloating objects per star is simply

$$
\frac{f_{\mathrm{HM}} \rho_{\text {halo }}}{n_{\star}}=10^{3} \mathrm{M}_{\oplus}\left(\frac{0.06}{\mathrm{f}_{\mathrm{HM}}}\right) .
$$

We also include three frequencies from other observational efforts:

1. Sumi et al. (2011) reported that there may be two freefloating Jupiter-mass planets per star in the MW. Although inconsistent with the (more recent) limits set by Mróz et al. (2017), we display this result for context.

2. Mróz et al. (2017) place an upper limit of fewer than 0.25 Jupiter-mass FFPs per star in the MW. This is 


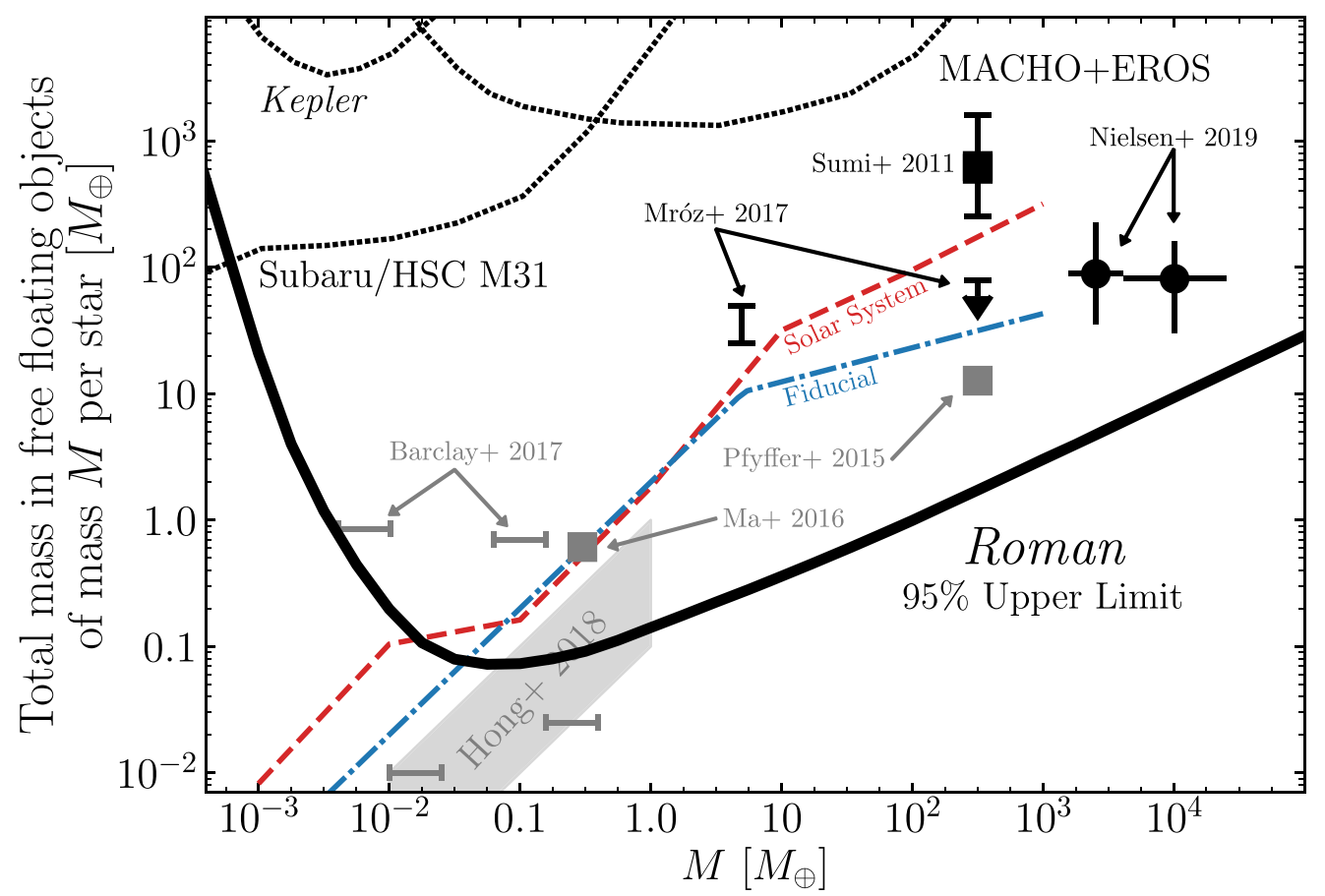

Figure 8. The thick solid black line shows the $95 \%$ confidence upper limit on the total mass of objects per star as a function of the object mass that Roman will be able to place if no objects of a fixed mass are detected. It is orders of magnitude lower than past limits and can test predictions on the abundance of FFPs from planetary formation (or free-floating compact objects formed from other mechanisms, such as primordial black holes). The black dashed lines represent similar limits placed by microlensing searches for massive compact halo objects. The blue dotted-dashed line shows our fiducial mass function (Equation (8)). For context, the red dashed line shows the case if roughly a solar system's worth of objects per star were free-floating in the Galaxy. The observational results of previous microlensing surveys are plotted in black points indicated by "Sumi +2011 " and "Mŕoz + 2017." The black circles are frequencies for widely separated bound planets reported by Nielsen et al. (2019) using direct imaging. Upper limits from three related studies are plotted in gray (see Section 4.2 for details). Citations: Alcock et al. (1996), Sumi et al. (2011), Griest et al. (2014), Ma et al. (2016), Barclay et al. (2017), Mróz et al. (2017), Hong et al. (2018), Niikura et al. (2019), Nielsen et al. (2019).

indicated by the black arrow in the upper right. Mróz et al. (2017) find a tentative signal for 5-10 $5 M_{\oplus}$ mass FFPs per star in the MW. This is represented by the black vertical bracket. Note that if no events occur with a Jupiter-mass lens, then Roman will place a limit of fewer than one Jupiter-mass planet per $\sim 100$ stars in the MW. This will improve the limit placed by the OGLE survey from $8 \mathrm{yr}$ of data by more than an order of magnitude (Mróz et al. 2017).

3. We consider measurements of the frequencies of bound planets and brown dwarfs found using direct imaging by Nielsen et al. (2019). While these are bound planet frequencies, they are for companions with semimajor axes from 10 to $100 \mathrm{au}$, which would likely be mistaken for free-floating planets in microlensing surveys, and thus provide a useful comparison. Nielsen et al. (2019) found a 3.5\% occurrence rate for 5-13 Jupiter-mass planets and a much lower rate of $0.8 \%$ for 13-80 Jupiter-mass brown dwarfs for hosts with mass $0.2>M / M_{\odot}>5$. We include these two frequencies as black circles in Figure 8, with vertical errors being their reported uncertainties and horizontal errors being the associated ranges. Roman will be sensitive to these widely bound companions, so distinguishing these free-floating planet false positives will be important.

We also plot predictions on the total mass of FFPs per star from a number of theoretical simulations:

1. Pfyffer et al. (2015) present simulations of formation and evolution of planetary systems, in which only $\sim 0.04 M_{\text {Jup }}$ of planets are ejected per star in the optimistic case of no eccentricity or inclination damping.

2. Ma et al. (2016) predict the number of planets ejected per star from dynamical simulations. We take values from their models of $0.3 M_{\odot}$ stars, in that $12.5 \%$ of stars eject $5 M_{\oplus}$ of mass in $0.3 M_{\oplus}$ bodies.

3. Barclay et al. (2017) predict the number of planetesimals ejected from systems during planet formation. We only compare our limit to their prediction in which giant planets are present in the system, as a gray horizontal bar spanning the width of the bins used. In the case that no giant planets are present, fewer objects are ejected.

4. Hong et al. (2018) predict that $\mathcal{O}(0.01-1)$ moons will be ejected from systems following planet-planet dynamical interactions. We assume that these moons have masses from 0.1 to $1 M_{\oplus}$, and thus a range of possibilities are included within the gray shaded region. This is a generous upper mass limit compared to the moons of our solar system, but we note that little is understood on the formation of exomoons. As an example of an unexpected possibility, there is (contested) evidence of a Neptune-sized exomoon in the Kepler-1625b system (Teachey \& Kipping 2018; Kreidberg et al. 2019; Teachey et al. 2020).

Thus, we conclude that Roman will not only improve the constraints on the abundance of objects with masses from that of less than the Moon to the mass of Jupiter by an order of magnitude or more but also allow for a test of model predictions for the total mass of ejected planets in several different planet formation and evolution theories. 


\section{Discussion}

\subsection{Event Detection}

The Roman microlensing survey will record nearly 40,000 photometric data points for $\sim 10^{8}$ stars over its $5 \mathrm{yr}$ duration. While we have perfect knowledge within these simulations, practically finding events due to very low mass lenses will likely require more sophisticated search algorithms. Microlensing surveys have used clear and specific cuts in identifying events. For example, Mróz et al. (2017) made a series of detection cuts based on the temporal distribution of data points during a candidate event, e.g., the number of observations obtained while the flux is rising and falling. These additional cuts were made in order to avoid false positives like flares or cataclysmic variables.

Machine-learning classifiers are also starting to be applied to microlensing survey data as well. Wyrzykowski et al. (2015) searched through OGLE-III data using a random forest classifier. Godines et al. (2019) present a classifier for finding events for low-cadence wide-field surveys. Khakpash et al. (2019) developed a fast, approximate algorithm for characterizing binary lens events. G. Bryden et al. (2020, in preparation) are developing a machine-learning classifier for the microlensing survey being performed with the United Kingdom Infrared Telescope (UKIRT; Shvartzvald et al. 2017). This survey is designed to be a pathfinder for the Roman survey and is mapping the near-infrared microlensing event rate in candidate Roman fields.

Still, most of these efforts have focused on the familiar regime of small source sizes $\left(\rho \sim 10^{-2}-10^{-3}\right)$ that are more familiar in microlensing surveys. It will need to be carefully examined how effective these search techniques are in detecting the extremely short timescale events we are considering, particularly those with qualitatively different morphologies from the more familiar $\rho \ll 1$ single lens microlensing events. Here we use only the $\Delta \chi^{2}$ and $n_{3 \sigma}$ metrics to determine whether an event is detected in these simulations (but see Appendix B). However, events may be detectable by Roman over a wider region of parameter space using different event selection filters, including the lowamplitude top-hat events caused by low-mass lenses.

\subsection{False Positives}

The full sample of microlensing events detected by Roman will need to be vetted for false positives. Detector artifacts such as hot pixels or other defects may introduce systematics that could mimic a short-timescale microlensing event. However, among the Core Community Surveys, the Galactic Exoplanet Survey provides one of the best opportunities to characterize the Wide Field Instrument H4RG detectors, facilitating the exclusion of these artifacts in light curves (Gaudi et al. 2019).

Astrophysical sources could also be mistaken for microlensing events. Similar to those false positives of ground-based microlensing surveys, these will include at least asteroids, cataclysmic variables, and flaring stars. While a more rigorous event detection algorithm will allow Roman to mitigate these in the full microlensing sample, even simple cuts will sometimes suffice. For example, M-dwarf flares rarely last longer than 90 minutes (Hawley et al. 2014), making their exclusion almost guaranteed by a simple cut on $n_{3 \sigma}$. Longer-lasting events such as novae or cataclysmic variables will have many photometric data points during their eruption to model and reject them.
Spatiotemporal clustering of candidate events and astrometric centroid analysis can be used to recognize asteroids that have not already been identified prior to a pipeline run.

\subsection{Degeneracies}

As identified in Mróz et al. (2017), one event (MOA-ip-01) in the sample of short-timescale events presented by Sumi et al. (2011) has a degenerate solution. The event was reported with $t_{\mathrm{E}}=0.73$ days, but an alternate solution with the much longer $t_{\mathrm{E}}=8.2$ days is favored. In this case, a larger blending parameter $\left(f_{s}\right)$ and smaller impact parameter $\left(u_{0}\right)$ resulted in the alternate solution. The major difference between these models is in the appearance of the wings of the magnification event. This degeneracy is well described in Woźniak \& Paczyński (1997). Roman should be able to distinguish between these approximately degenerate events via its high precision and cadence.

Another relevant degeneracy occurs in lensing events with large relative angular source size $\rho$. In this regime, the magnification over the duration of the event is roughly constant (in the absence of limb darkening) and set by Equation (A2). As a result, $\rho$ becomes nearly degenerate with the blending parameter $f_{\mathrm{S}}=F_{\mathrm{S}} /\left(F_{\mathrm{S}}+F_{\mathrm{B}}\right)$, which is the fraction the source flux $F_{\mathrm{S}}$ contributes to the observed baseline flux $F_{\mathrm{S}}+F_{\mathrm{B}}$, where $F_{\mathrm{B}}$ is the blended flux. Essentially, the flux from the source alone cannot be confidently measured without precise and dense photometry, which can be used to distinguish the subtle differences in these broadly degenerate light curves. Thus, in the presence of blended light, one may underestimate the true peak magnification (S. A. Johnson et al. 2020, in preparation; Mróz et al. 2020), making it difficult to constrain $\rho$ precisely. This is important because $\rho$ depends on the angular source size, which will be poorly constrained when no color measurement is made while the source is magnified. These measurements will likely not occur for short-timescale events and those that exhibit extreme finite-source effects. We note that this degeneracy persists even in the presence of limb darkening (S. A. Johnson et al. 2020, in preparation)

For fixed $\theta_{*}, \rho$ increases as the planet mass (and thus $\theta_{\mathrm{E}}$ ) decreases. Thus, as the lens mass decreases, more and more events enter into the $\rho \gg 1$ regime, thereby increasing the likelihood that they will suffer from this degeneracy. We note that this continuous degeneracy is different from the discrete degeneracy exhibited in the event reported by Chung et al. (2017).

In order to estimate the fraction of events for which finitesource effects should be detectable, in Figure 9 we show the cumulative fraction of detected events as a function of $\rho / u_{0}$. Events that have $\rho / u_{0}=\theta_{*} / \theta_{0} \gtrsim 0.5$ should exhibit finitesource effects (Gould \& Gaucherel 1997). Events that satisfy this criterion and have $\rho \gg 1$ will be more susceptible to the above $\rho-f_{\mathrm{S}}$ degeneracy.

Fortunately, most of our low-mass lenses that exhibit finitesource effects will be detected in events where the source star dominates the baseline flux (or have large values of $f_{\mathrm{S}}$ ). This is shown in Figure 10, where we plot the cumulative fraction of detected events as a function of $f_{\mathrm{S}}$ in W146 (top panel) and Z087 (bottom panel). Vertical dashes mark the median values of $f_{\mathrm{S}}$ of these distributions, and note that the markers for $10^{2}$ and $10^{3} M_{\oplus}$ lie on top of each other. We also include the source magnitude distributions for detected events in Figure 11 for W146 (top panel) and Z087 (bottom panel). Brighter sources 


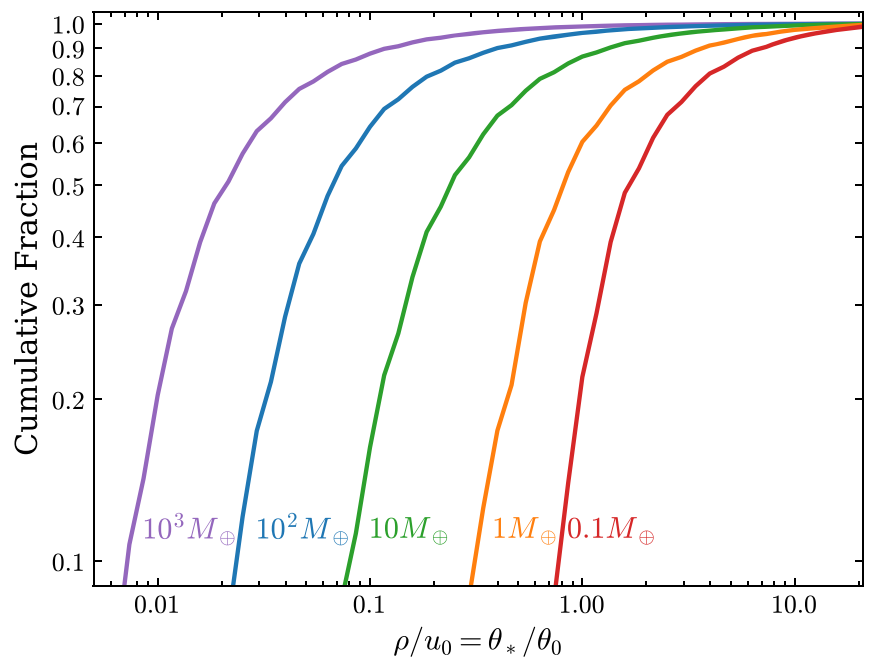

Figure 9. Cumulative fraction of detected events as a function of $\rho / u_{0}$, which is equal to $\theta_{*} / \theta_{0}$, or the angular source radius relative to the angular impact parameter. Almost all low-mass lens events detected will exhibit finite-source effects (with $\rho / u_{0} \gtrsim 0.5$; Gould \& Gaucherel 1997).

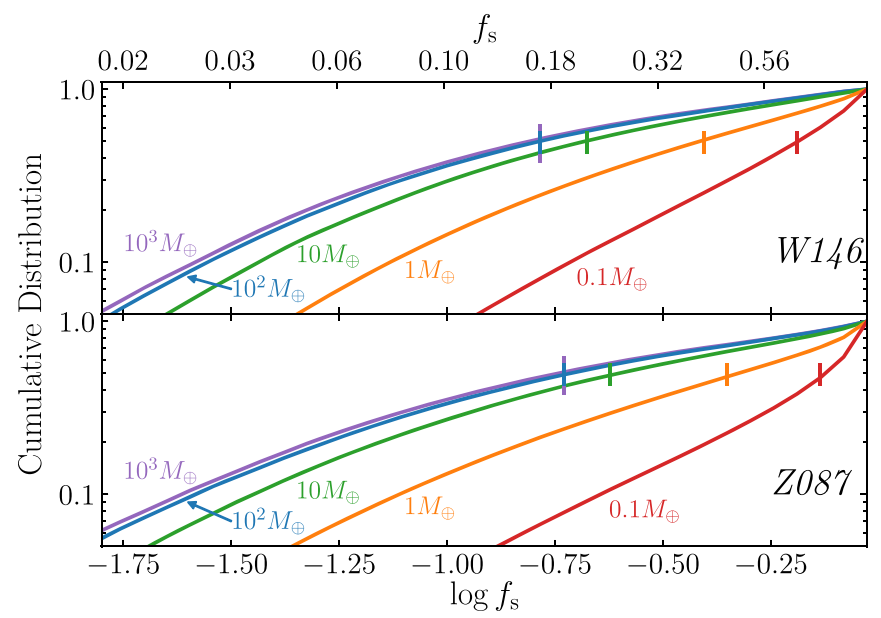

Figure 10. Most source stars will contribute the majority of baseline flux in low-mass lens events. Here we show normalized cumulative distributions of the blending parameter for detected events among the five mass bins. For each lens mass, a vertical tick on the distribution marks the value of $f_{s}$ at which half of events have a greater $f_{s}$. For higher-mass lenses, this value is $f_{s} \approx 0.20$, and this value only increases as lens mass decreases. For the $0.1 M_{\oplus}$ lenses, most detected events have $f_{s}>0.5$, and thus the source makes up the majority of the baseline flux. Top: blending in W146. Bottom: blending in Z087.

will contribute most to the low-mass lens event rates, as one would expect. However, because the fraction of blended flux is not known a priori, this argument can only be used in a statistical sense.

Events of all masses will have little blending in Z087, but small mass lenses (that last $\lesssim 6 \mathrm{hr}$ ) will be unlikely to have a Z087 measurement taken while magnified. Measurements from multiple filters may allow an estimate of the source color. Table 3 shows the fraction of events that will have a color measurement taken while the source is magnified, resulting in the breaking of the degeneracy. Only $11 \%$ of $0.1 M_{\oplus}$ lenses will have a color measurement if the $Z 087$ measurements (or other alternative band) have a cadence of $12 \mathrm{hr}$, but this fraction can be more than tripled if the cadence increases to $6 \mathrm{hr}$. At $1.0 M_{\oplus}$, the

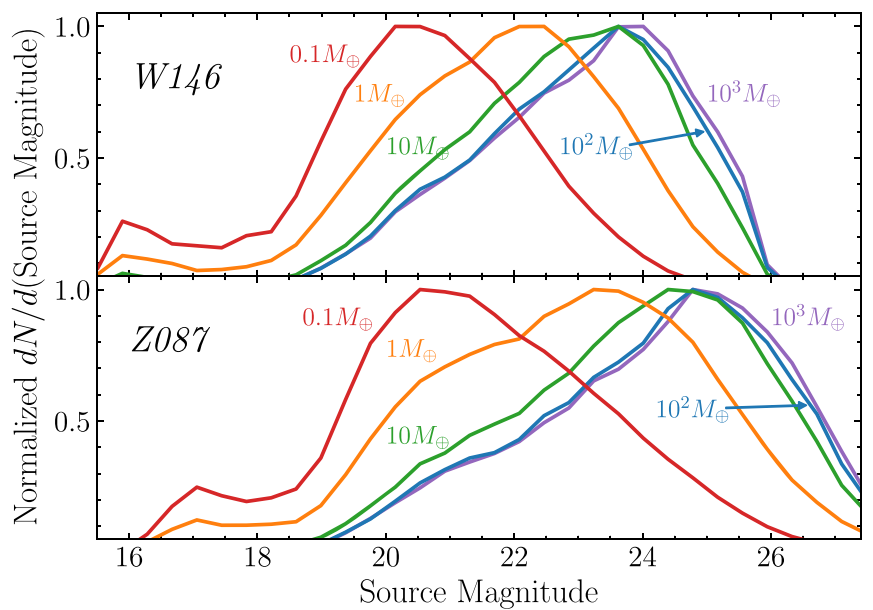

Figure 11. Low-mass lenses will have much brighter source stars. Here we show the source magnitude distributions normalized to their peaks. The highermass lenses $\left(\gtrsim 10 M_{\oplus}\right)$ will have nearly identical distributions, but lower masses than that will strongly deviate. Top: source magnitude distribution in W146. Bottom: source magnitude distribution in Z087.

Table 3

Fraction of Detected Events with Color Measurements While Source Is Magnified

\begin{tabular}{lccccccc}
\hline \hline \multirow{2}{*}{$\begin{array}{l}\text { Mass } \\
\left(M_{\oplus}\right)\end{array}$} & $12 \mathrm{hr}$ & $6 \mathrm{hr}$ & $3 \mathrm{hr}$ & & & \multicolumn{3}{c}{$n_{3 \sigma} \geqslant 3 \mathrm{hr}$} & $6 \mathrm{hr}$ & $3 \mathrm{hr}$ \\
\hline 0.1 & $11 \%$ & $35 \%$ & $56 \%$ & & $8 \%$ & $25 \%$ & $42 \%$ \\
1.0 & $12 \%$ & $23 \%$ & $37 \%$ & & $11 \%$ & $20 \%$ & $34 \%$ \\
10 & $32 \%$ & $53 \%$ & $75 \%$ & & $32 \%$ & $53 \%$ & $75 \%$ \\
100 & $74 \%$ & $92 \%$ & $96 \%$ & & $75 \%$ & $93 \%$ & $96 \%$ \\
1000 & $98 \%$ & $99 \%$ & $99 \%$ & & $98 \%$ & $99 \%$ & $99 \%$ \\
\hline
\end{tabular}

fraction of detected events with a color measurement will double if the color cadence increases to $6 \mathrm{hr}$. We also include fractions of detected events with color measurements if our threshold $n_{3 \sigma} \geqslant 6$ were to be relaxed to only 3 , making the percentage even lower for low-mass lenses. Note that the modest decrease in percentages arises from the fact that many more low-mass lens events are detected when the $n_{3 \sigma}$ threshold is relaxed (see Appendix B, especially Figure B2).

Alternatively, this degeneracy may be broken through the 5 yr baseline of the microlensing survey. Potentially blended sources may become apparent as blended stars (either unrelated stars, the host star if the planet is actually bound but widely separated (see Section 5.4), or a companion to the host star) move away from the line of sight to the source. This fact will also be used in constraining the presence of potential host stars to FFP candidates.

S. A. Johnson et al. (2020, in preparation) demonstrate that there is a second degeneracy in events with $\rho \gg 1$. This is a multiparameter degeneracy between the effective timescale of the event, which is well approximated by the time to cross the chord of the source $t_{c}$, the impact parameter of the lens with respect to the center of the source $u_{0, *}$, and the time to cross the angular source radius $t_{*}=\theta_{*} / \mu_{\text {rel }}$ (see Appendix $\mathrm{A}$ for definitions of these quantities). This is easiest to understand in the absence of limb darkening. A larger impact parameter $u_{0, *}$ results in a shorter event, but with the same peak 
magnification (due to the "top-hat" nature of events with $\rho \gg 1)$. But this shorter duration can be accommodated by scaling $t_{*}$. Since neither $\theta_{*}$ nor $\mu_{\text {rel }}$ is known a priori, it is impossible to measure $\mu_{\text {rel }}$ in the regime where these assumptions hold. S. A. Johnson et al. (2020, in preparation) demonstrate that this degeneracy holds for limb-darkened sources as well.

We are investigating the severity and impact of these degeneracies on the ability to recover event parameters in events with extreme finite-source effects (S. A. Johnson et al. 2020 , in preparation).

\subsection{Wide-bound Confusion}

While Roman will have sensitivity to the short-timescale events of FFPs, true FFPs can be confused with widely separated but bound planets. If a bound planet has a large enough projected separation, the source may only be magnified by the planet and not the host star (Di Stefano \& Scalzo 1999; Han \& Kang 2003; Han et al. 2005). This confusion requires proper accounting if accurate occurrence rates for both FFPs and wide-bound planets are to be reached. To this end, Han et al. (2005) summarize three methods for distinguishing the presence of a host star.

The first method was originally described by Han \& Kang (2003), in which the magnification by a bound planetary-mass object will deviate from that of an isolated lens. In this scenario, rather than the effective point caustic of an isolated lens, the source is magnified by a planetary caustic that changes the morphology of the light curve. Han \& Kang (2003) assume some fiducial detection thresholds and find that this method can distinguish $\gtrsim 80 \%$ of events for projected separations $\lesssim 10$ au and mass ratios down to $q \approx 10^{-4}$. This deviation was first observed by Bennett et al. (2012) and more recently observed in the short (4-day) event reported by Han et al. (2020b), where the presence of a host was determined through $0.03 \mathrm{mag}$ residuals near the peak magnification of a single lens model.

Another pathway to determine whether an FFP lens is truly isolated would be to rule out any magnification from a photometrically undetected host (Han et al. 2005). This signal would appear as a long-term, low-amplitude bump in the light curve. Han et al. (2005) show that nearly all planets with projected separations of less than about 13 au will have the presence of their host stars inferred this way. Assuming that the semimajor axis distribution of bound planets is log-uniform, $\sim 30 \%$ of those with $a \in\left[10^{-1}, 10^{2}\right]$ lie outside 13 au.

The third method is to directly measure blended light from a candidate host. This can be performed in earlier or later seasons with Roman by searching for PSF elongation, color-dependent centroid shifts, or event resolution of the lens host star and the source star. Henderson \& Shvartzvald (2016) find that Roman can exclude hosts down to $\gtrsim 0.1 M_{\odot}$ depending on the lens distance and the nature of the source star. Paper I finds that the majority of hosts to bound planet detections will contribute at least $10 \%$ of the total blend flux. The separation of unassociated blended stars (neither the lens nor source star) from potential host flux could be constrained through priors from the event, such as the distance to the lens system.

\subsection{Limb Darkening and Wave Optics}

We did not account for the effects of limb darkening in our simulations (Witt 1995; Heyrovský 2003). The limb-darkening profile of source stars is wavelength dependent, with the amplitude of the surface-to-limb variation decreasing $\propto \lambda^{-1}$ for the Sun (Hestroffer \& Magnan 1998). Because the primary observations are in the near-infrared, Roman typical source stars will exhibit less limb darkening than in optical surveys. As shown by Lee et al. (2009) and many more, the limbdarkening profile alters the shape of the light curve (see their Figure 6). This would likely impact our yield estimates for lowmass lenses most, where finite-source effects are most likely. In our detection cuts, if a source is fainter in its limb, it may shorten the effective timescale of the event. This could lower $n_{3 \sigma}$ or $\Delta \chi^{2}$ of the event in our detection threshold. However, limb darkening increases the peak magnification of events (see bottom panel of Figure 2), which could modestly increase the number of detections we predict. We must also consider that W146 is a wide band, and thus the limb darkening will have a significant chromatic dependence over the wavelength range of the filter (Han et al. 2000; Heyrovský 2003; Claret \& Bloemen 2011).

If the mass of a lens is small enough $\left(\sim 10^{-5} M_{\oplus}\right)$, the geometric optics description of microlensing becomes insufficient and wave effects manifest themselves in the magnification curve (Takahashi \& Nakamura 2003, among others). In short, the threshold for this effect is when the wavelength of light being observed becomes comparable to the Schwarzschild radius of the lens; in this limit there is a fundamental limit to the peak magnification of the event. For a mass of $10^{-3} M_{\oplus}$ $\left(3 \times 10^{-9} M_{\odot}\right)$ this corresponds to a wavelength of $\sim 11 \mu \mathrm{m}$ (see Equations (5) and (7) of Sugiyama et al. (2020)). This is below the long-wavelength edge of the W146 band, and the corresponding wavelength only gets longer for larger mass lenses. We therefore do not consider this effect here.

\subsection{Mass Measurements}

The conversion from timescale to mass for FFP events requires measurements of both the microlensing parallax and the angular Einstein ring. A measurement of $\rho$ from finitesource effects and $\theta_{*}$ from the dereddened source flux and colors (Yoo et al. 2004) would yield $\theta_{\mathrm{E}}$, if the degeneracy discussed above can be broken and measurements are made in another filter(s) while the source is magnified. ${ }^{11}$

Spitzer enabled the regular measurement of microlensing parallaxes to a large number of stellar, binary, and boundplanetary microlensing events by leveraging the fact that it was separated by the Earth by $\sim 1$ au owing to its Earth-trailing orbit (Gould 1994, 1995, 1999), but these events had projected Einstein ring sizes of a few au (e.g., Dong et al. 2007; Yee et al.

2015). Zhu \& Gould (2016) quantify the potential for simultaneous ground-based observations (and Roman-only observations) to measure one- and two-dimensional microlens parallaxes. Space-based parallax measurements of FFP lenses were also attempted using the Kepler spacecraft during the K2 Campaign 9 survey, which largely consisted of a microlensing survey toward the bulge (Henderson \& Shvartzvald 2016; Henderson et al. 2016; Zhu et al. 2017a, 2017b; Penny et al. 2017; Zang et al. 2018). Penny et al. (2019) and Bachelet \&

\footnotetext{
11 We note that typically the empirical relations used to convert from source flux and color to angular radius are based on measurements from giant stars, which are most likely to exhibit finite-source effects in microlensing events (e.g., van Belle 1999). For lenses with low enough masses, however, we will need appropriately calibrated relations for nongiant source stars (e.g., Adams et al. 2018).
} 
Penny (2019) show that the short intra-L2 baseline between the Euclid and Roman spacecraft would be enough to measure free-floating planet parallaxes. Ban (2020) computes probabilities for measuring parallaxes for combinations of ground- and space-based telescopes. Concurrent observations with widefield infrared observatories, such as UKIRT (Hodapp et al. 2018), VISTA (Dalton et al. 2006), and PRIME (Yee et al. 2018), ${ }^{12}$ as well as wide-field optical observatories, such as DECam (Flaugher et al. 2015), HyperSuprimeCam (Miyazaki et al. 2012), and the Vera C. Rubin Observatory (LSST Science Collaboration et al. 2009), would enable parallax measurements for both bound and free-floating planets.

\section{Conclusion}

We have used GULLS simulation software (Penny et al. 2019) to show that the Roman Galactic Exoplanet Survey will inform our understanding of the isolated compact object mass function throughout the Galaxy, down to very low planetarymass objects. In particular, it will be able to detect microlensing events with timescales as short as $1.5 \mathrm{hr}$, and thus isolated lenses with masses down to at least $0.1 M_{\oplus}$. This data set will be used to address questions about both the low-mass tail of the initial mass function of stars and the total mass and mass function of objects ejected from planetary systems during planet formation and evolution. Roman will be able to probe populations of free-floating planets that are essentially impossible to access from ground-based microlensing surveys. Finally, the limits that Roman will place if no such objects are detected would be the most stringent to date by orders of magnitude.

We are particularly proud to honor Nancy Grace Roman, after whom this survey telescope has recently been named. We hope to live up to her extraordinary influence on space astronomy.

We appreciate the revisions from the referee that improved the quality of this work, as well as those from careful readings by Radek Poleski and Przemek Mróz. We thank our colleagues Andrew Gould and David Bennett for useful discussions. We thank everyone on the Roman Galactic Exoplanet Survey Science Investigation Team. We also appreciate Exoplanet Lunch at Ohio State University, which was the source of many useful discussions. S.A.J. dedicates his contribution to this work to David John Prahl Will, without whom this work and that of many others would not be possible.

This work was performed in part under contract with the California Institute of Technology (Caltech)/Jet Propulsion Laboratory (JPL) funded by NASA through the Sagan Fellowship Program executed by the NASA Exoplanet Science Institute. S.A.J., M.T.P., and B.S.G. were supported by NASA grant NNG16PJ32C and the Thomas Jefferson Chair for Discovery and Space Exploration.

Software: astropy (Astropy Collaboration et al. 2013, 2018), Matplotlib (Hunter 2007), MulensModel (Poleski \& Yee 2018), VBBinaryLensing (Bozza 2010; Bozza et al. 2018).

\footnotetext{
12 https://www.astro.caltech.edu/ srk/Workshops/TDAMMS / Files4Facilities/PRIME.pdf
}

\section{Appendix A \\ An Introduction to Microlensing in the Large Angular Source Regime}

\section{A.1. Light-curve Morphology}

For a typical isolated microlens, the angular size of the source is much smaller than the angular size of the Einstein ring of the lens, and thus the approximation of a point source generally remains valid. That is, the magnification as a function of the separation between the source and the lens normalized to the size of the Einstein ring $u$ is given by (Paczyński 1986)

$$
A=\frac{u^{2}+2}{u\left(u^{2}+4\right)^{1 / 2}} .
$$

The magnification peaks at the minimum separation $u_{0}=\theta_{0} / \theta_{\mathrm{E}}$, where $\theta_{0}$ is the angular separation between the source and the lens at closest approach. The point-source approximation in Equation (A1) breaks down when angular separation of the source from the lens, $\theta_{0}$, becomes comparable to the angular radius of the source $\operatorname{star} \theta_{*}$. For point lenses, this condition results in a significant second derivative in the pointlens light curve over the angular size of the source, which must be accounted for by computing the magnification. Thus, for events with impact parameter such that $\rho / u_{0} \gtrsim 0.5$, where $\rho=\theta_{*} / \theta_{\mathrm{E}}$, the peak of the event (at times $\left|t-t_{0}\right| / t_{\mathrm{E}} \lesssim 2 \rho$ ) is affected by finite-source effects (Gould \& Gaucherel 1997). Since, for stellar mass lenses, $\rho$ is typically in the range of $10^{-3}$ to $10^{-2}$, most events are unaffected, and those that are affected are high-magnification events. Even in such events, finitesource effects are only detectable near the peak of the event, while the magnification during the rest of the event is essentially equivalent to that due to a point source.

However, this characterization breaks completely when the angular size of the source becomes comparable to the angular size of the Einstein ring, or $\rho \gtrsim 1$. In particular, in the extreme case when $\rho \gg 1$, the source will completely envelop the Einstein ring of the lens if it passes within the angular source radius. When this happens, the lens magnifies only a fraction of the area of the source as it transits the disk of the source (e.g., Gould \& Gaucherel 1997; Agol 2003). In this limit $(\rho \gg 1)$ and to first order, the magnification curve can take on a "top-hat" or boxcar shape, saturating at a magnification of

$$
A_{\text {peak }} \approx 1+\frac{2}{\rho^{2}}
$$

(Liebes 1964; Gould \& Gaucherel 1997; Agol 2003). The lightcurve shape is essentially independent of $u_{0}$ except when $u_{0} \sim \rho$ (Agol 2003). Furthermore, the duration of the event is no longer set by the microlensing timescale $t_{\mathrm{E}}$, but rather is proportional to the source radius crossing time $t_{*}=\theta_{*} / \mu_{\text {rel }}=t_{\mathrm{E}} \rho$. Mróz et al. (2018) account for the impact parameter $u_{0}$ such that they use the time taken for the lens to cross the chord of the source

$$
t_{c}=\frac{2 \theta_{*}}{\mu_{\mathrm{rel}}} \sqrt{1-\left(u_{0, *}\right)^{2}},
$$

where $u_{0, *}=\theta_{0} / \theta_{*}$. Note that this timescale is independent of the angular radius of the Einstein ring and thus lens mass. We note that Mróz et al. (2017) define $t_{*}$ as the crossing time over 


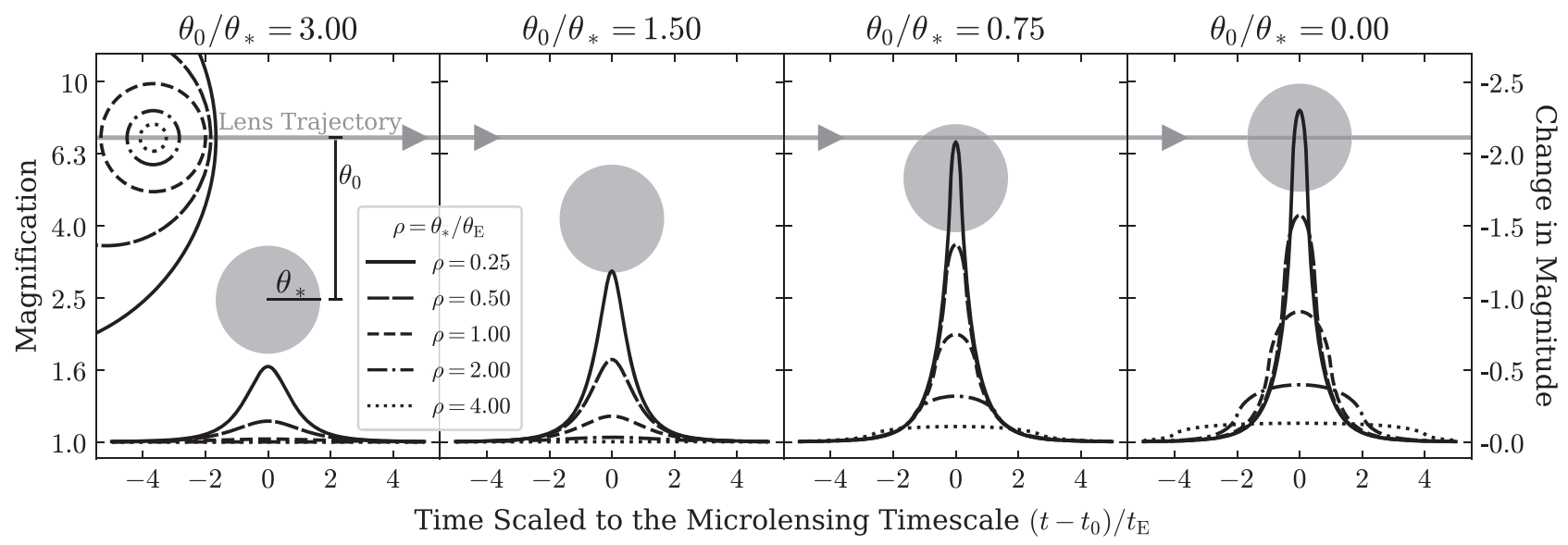

Figure A1. The morphology of microlensing light curves changes as finite-source effects become more prominent. In the background, we show a gray circle that represents the source (with an angular radius $\theta_{*}$ ). We also show five Einstein rings scaled to the source size, which have $\rho=\theta_{*} / \theta_{\mathrm{E}}$ values indicated in the legend. Each panel is for a different impact parameter $u_{0, *}=3.00,1.50,1.00$, and 0.00 from left to right. We change the scaled position of the source star circle relative to the lens trajectory (gray horizontal line) to match the impact parameter. For each panel, we plot the magnification as a function of time scaled to the microlensing timescale $\left(t-t_{0}\right) / t_{\mathrm{E}}$. For the most extreme case of $\rho=4.00$, we see no appreciable magnification until the lens traverses the source $\left(u_{0, *}<1\right)$, at which point the magnification is essentially constant (except when the lens is near the edges of the source). The light curves thus have a "top-hat" appearance. We note that this "tophat" morphology only appears when there is no limb darkening. All events have peak magnifications that monotonically increase as $u_{*}$ decreases; however, this maximum magnification begins to saturate at the expected value of $1+\frac{2}{\rho^{2}}$ for $\rho>1$. However, the length of those events with $\rho>1$ is significantly longer than expected from their analytic $t_{\mathrm{E}}$ timescales.

the chord of the source, but we define $t_{*}$ as the source radius crossing time defined above (see Appendix A of Skowron et al. 2011). Henceforth, we will use $t_{c}$ for the chord-crossing time, and we propose that this become the convention.

Broadly, these changes in light-curve morphology are referred to as extreme finite-source effects, as the light curve is affected by finite-source effects throughout the duration (e.g., at no time while the source is magnified does the point-source approximation hold). We demonstrate the impact of finitesource effects on the light curve of events in Figure A1. We consider five lenses in which we only vary the angular size of the Einstein ring, quantified by $\rho=0.25,0.50,1.00,2.00$, and 4.00 (as the size of the source is fixed). The sizes of these rings are shown in the upper left corner of the leftmost panel scaled to the size of a source star, which is depicted as a gray circle (with an angular radius of $\theta_{*}$ ). A horizontal gray line depicts the path the lenses will take, and it is separated from the center of the source star by the impact parameter $\theta_{*}$ (again, to scale). We then vary the impact parameter from $u_{0, *}=3.0$ down to $u_{0, *}=0.0$ from left to right. This is written above the plot and depicted as the source star (gray circle) approaching the lens trajectory (gray line). Note that the circles and their separations are independent of the time and magnification axes.

Each panel depicts all five lenses in different events, where the line style matches the lens with the same Einstein ring line style in the upper left corner of the leftmost panel. We use the method of Lee et al. (2009) as implemented in MulensModel (Poleski \& Yee 2018) to compute all light curves in this figure.

As $\theta_{\mathrm{E}} \propto \sqrt{M}$, the more massive lens will have the largest $\theta_{\mathrm{E}}$ and be the farthest from the regime of finite-source effects. Note that time is referenced to the peak of the event $\left(t_{0}\right)$ and scaled by the analytic timescale $t_{\mathrm{E}}$ on the horizontal axis. The solid-line light curve behaves essentially how you expect an isolated lens to behave as the impact parameter drops up until the last two panels where the peak becomes more rounded. This is the first breakdown we described that occurs when $\rho / u_{0} \gtrsim 0.5$, or when the size of the source star is within a few times the impact parameter.
However, the behavior is dramatically different for the lowest-mass lens $(\rho \gg 1)$. In this case, when lens is not transiting the source $\left(u_{*}>1\right)$, there is effectively no magnification. For the smallest impact parameter (rightmost panel), the light curve looks like the top hat described earlier, magnifying the source by roughly $10 \%\left(1+\frac{2}{4^{2}} \approx 1.13\right)$. Also note that the duration of this event is now much longer than one would expect given its analytic timescale $t_{\mathrm{E}}$. In fact, when $u_{0, *}=0.00$, the duration is nearly exactly what we predict given the diameter crossing time $t_{c} / t_{\mathrm{E}}=2 \rho=4$ for the $\rho=2$ case and $t_{c} / t_{\mathrm{E}}=8$ for the $\rho=4$ case. In the rightmost panel, the $\rho=4$ event lasts $\sim 4$ times longer than one would expect based on the value of $t_{\mathrm{E}}$.

To provide a quantitative sense of the relevant scales, consider a typical stellar mass lens $\left(0.3 M_{\odot}\right)$, which has an angular Einstein ring radius of $\theta_{\mathrm{E}}=550 \mu$ as. A source star in the Galactic bulge (at a distance of $D_{\mathrm{S}}=8 \mathrm{kpc}$ ) that has a radius of $1 R_{\odot}$ will have an angular radius of just $0.6 \mu$ as. Lenses with mass $\lesssim 0.12 M_{\oplus}$ will have $\rho \gtrsim 1$ for this source. A typical clump giant in the bulge will have a radius of $\sim 10 R_{\odot}$, leading to $\rho>1$ for lenses with mass $\lesssim 10 M_{\oplus}$.

These morphological changes will impact the microlensing event rate and microlensing optical depth (Vietri \& Ostriker 1983; Paczyński 1991). Recall that the microlensing optical depth (the probability that any given star is being lensed) is a function of the fraction of the sky covered by Einstein rings. As demonstrated above, lenses with small enough masses will have Einstein rings smaller than the angular size of some stars. Han et al. (2005) show that for lenses with low enough masses, the event rate actually increases compared to what you would expect for lenses with Einstein rings smaller than the angular size of source stars. For these lenses, the event rate is proportional to the fraction of the sky covered by source stars. However, the detection of such events is hampered by the fact that the peak magnification is lower than one would expect for a point source. Han et al. (2005) also derive analytic expressions for the threshold impact parameter for detection 
and the minimum detectable mass lens as function of the threshold signal-to-noise ratio for detection.

In reality, the shape of the light curve is sensitive to the limbdarkening profile of the source, as well as any of its surface features (e.g., Witt \& Mao 1994; Gould \& Welch 1996; Agol 2003; Heyrovský 2003; Yoo et al. 2004; Lee et al. 2009). The impact of included limb darkening is shown in the bottom panel of Figure 2. The "top-hat" shape disappears, and the light curve becomes more rounded. The example in Figure 2 adopted a single-parameter linear limb-darkening profile, but more structure could be added if a more complex profile was used (e.g., Claret \& Bloemen 2011), or if surface features (such as starspots) were considered (Heyrovský 2003).

\section{Appendix B Detection Criteria}

We require that simulated events pass two criteria to qualify as detections. The first is based on the deviation $\left(\Delta \chi^{2}\right)$ the event causes from a flat light curve

$$
\Delta \chi^{2}=\chi_{\text {Line }}^{2}-\chi_{\text {FSPL }}^{2}
$$

where $\chi_{\text {Line }}^{2}$ is the $\chi^{2}$ value of the simulated light curve for a flat line at the baseline flux and $\chi_{\mathrm{FSPL}}^{2}$ is the same but for the simulated data to the injected finite-source point-lens model of the event. The second criterion is the number of consecutive data points that are measured at $3 \sigma$. In this section we isolate the effect of the value of each criterion on the yield as a function of planet mass in turn, and then we consider the complex interplay between them.

We first plot the cumulative number of detected events $N_{\text {det }}\left(X \geqslant \Delta \chi^{2}\right)$ as a function of the threshold $\Delta \chi^{2}$ in Figure B1. We show the cases of our five discrete masses under the assumption that there is one such planet per star. Applying only this criterion, we can analytically estimate the impact of mission/survey design changes on the yield of FFPs by inferring the impact those changes would have on the $\Delta \chi^{2}$ of events (akin to Paper I). This is because the distributions in Figure B1 can be locally fit by a power law

$$
N\left(\Delta \chi^{2}>X\right) \propto X^{\alpha},
$$

over a wide range of $\Delta \chi^{2}$, as has previously been shown by Bennett \& Rhie (2002). We fit this power law for each mass in the range $\Delta \chi^{2}=[150,600]$ and list the values of exponents in Table B1.

While we necessarily employ a $\Delta \chi^{2} \geqslant 300$ as one of our thresholds, basing detection rates solely on this criterion is problematic because of the potential for very short events, e.g., events with only a few extremely magnified points that together contribute more than 300 to the $\Delta \chi^{2}$. Modeling these events would be challenging. We therefore also impose the second criterion on $n_{3 \sigma}$, which is specifically the number of consecutive data points with $n>3 \sigma$ above the baseline flux of the source star plus blend. ${ }^{13}$ This criterion ensures that there will be a sufficient number of high signal-to-noise ratio data points during the events that they can be confidently modeled.

To illustrate how this criterion changes the cumulative number of detections relative to just applying the $\Delta \chi^{2}$ criterion, in Figure B1 we plot as short-dashed (long-dashed)

\footnotetext{
13 We note that similar criteria were used by Sumi et al. (2011) and Mróz et al. (2017).
}

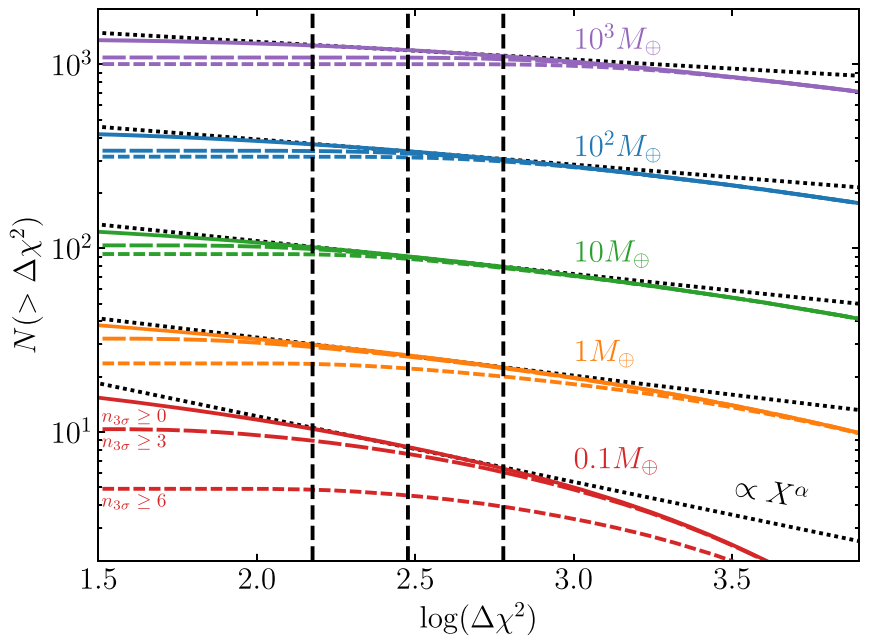

Figure B1. Cumulative distribution of the $\Delta \chi^{2}$ of simulated events. From bottom (red) to top (purple), the solid lines represent yields of lenses with masses of 0.1 to $10^{3} M_{\oplus}$, assuming one FFP of that mass per star in the MW. From left to right, the vertical black dashed lines indicate where $\Delta \chi^{2}=150$, $\Delta \chi^{2}=300$, and $\Delta \chi^{2}=600$. We fit a power law (Equation (B2)) to each of the solid lines for a range of $\Delta \chi^{2} \in[150,600]$. The slopes of these are included in Table B1. We also plot the cumulative $\Delta \chi^{2}$ distributions for events with $n_{3 \sigma} \geqslant 3$ and $\geqslant 6$ as the long-dashed and short-dashed lines, respectively. The distributions flatten significantly for lower $\Delta \chi^{2}$ when we require $n_{3 \sigma} \geqslant 6$.

Table B1

Slopes of $\Delta \chi^{2}$ Distributions

\begin{tabular}{lccc}
\hline \hline$M\left(M_{\oplus}\right)$ & \multicolumn{3}{c}{$\alpha$} \\
\cline { 2 - 4 } & $n_{3 \sigma} \geqslant 0$ & $n_{3 \sigma} \geqslant 3$ & $n_{3 \sigma} \geqslant 6$ \\
\hline 0.1 & -0.36 & -0.28 & -0.15 \\
1 & -0.21 & -0.19 & -0.12 \\
10 & -0.18 & -0.17 & -0.13 \\
100 & -0.14 & -0.080 & -0.040 \\
1000 & -0.097 & -0.013 & -0.0020 \\
\hline
\end{tabular}

lines the distributions also requiring $n_{3 \sigma} \geqslant 6\left(n_{3 \sigma} \geqslant 3\right)$ points. We fit the slopes of the cumulative distributions as power-law distributions as before over the same range, and we include the power-law exponents in Table B1. For a given mass, the distributions we derive applying both criteria change relative to only applying the $\Delta \chi^{2}$ criterion in a manner that depends on the mass of the lens.

We note that, for all of the masses, the cumulative distributions begin to fall below the power-law fit to the solid curves (without the $n_{3 \sigma}$ cut) at the highest values of the threshold $\Delta \chi^{2}$. Furthermore, the onset of this deviation occurs for lower values of $\Delta \chi^{2}$ for the very smallest masses. This deviation is due to the onset of finite-source effects and the increasing importance of these effects for lower masses.

Conversely, for lower values of the threshold $\Delta \chi^{2}$, the cumulative distributions begin to fall below the power-law fit to the solid curves at roughly the same value of $\Delta \chi^{2}$ for the three largest masses, but at different values for the lowest two masses. Finally, for all the masses, the cumulative distribution of the number of detections becomes essentially flat (independent of the $\Delta \chi^{2}$ threshold) for values of $\Delta \chi^{2} \lesssim 150$ and $n_{3 \sigma} \geqslant 6$. Thus, for this combination of detection criteria, the yield does not improve with a lower $\Delta \chi^{2}$ threshold, only with changing the $n_{3 \sigma}$ cut. These behaviors are all consistent with 


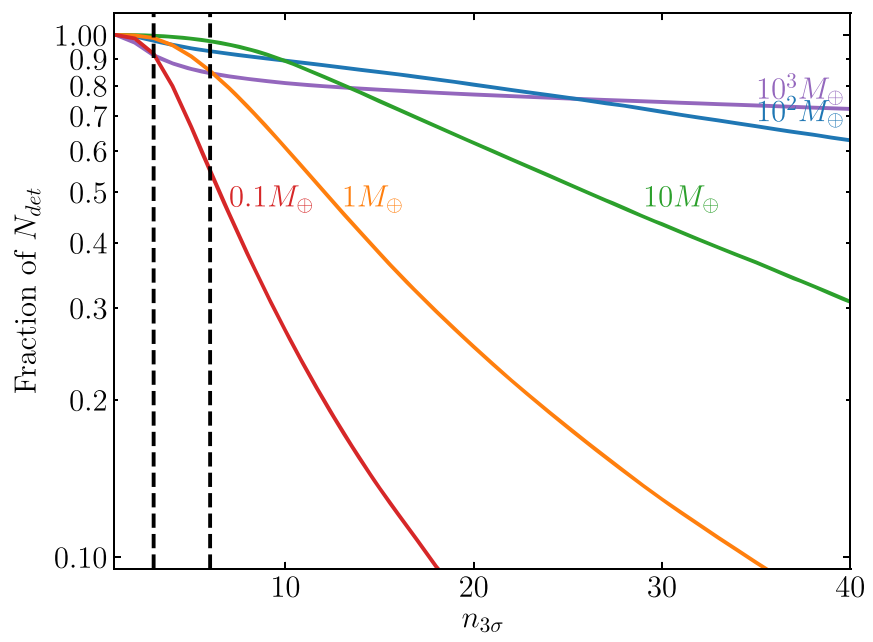

Figure B2. Cumulative fraction of events as a function of $n_{3 \sigma}$ with $\Delta \chi^{2}>300$. Each line represents only events with the labeled mass. The left (right) vertical dashed lines are at $n_{3 \sigma}=3\left(n_{3 \sigma}=6\right)$. The most significant difference between these thresholds is for the yields of $0.1 M_{\oplus}$ FFPs, which nearly doubles when the threshold is relaxed. These events are typically short or have low magnification. For masses above $10 M_{\oplus}$, the number of detections is relatively independent over the range $6<n_{3 \sigma}<40$. We conclude that the impact of our $n_{3 \sigma}$ detection criterion is highest for low-mass lensing events.

expectations based on the gradual change in the morphologies of the light curves as finite-source effects begin to dominate (roughly for masses $\lesssim M_{\oplus}$; see Appendix A).

To further explore the interplay between the two detection criteria, we isolate the effect of the $n_{3 \sigma}$ cut on the yields in Figure B2. Here we show the cumulative fraction of events as a function of $n_{3 \sigma}$ for events with $\Delta \chi^{2} \geqslant 300$.

For the two largest masses, the yield is a relatively weak function of $n_{3 \sigma}$ since these masses typically give rise to longertimescale (and thus more well-sampled) events. Interestingly, we find that $10 M_{\oplus}$ events are the most robust to this selection criterion for $n_{3 \sigma} \lesssim 10$; however, it falls off quickly afterward, as expected. The lowest two masses continue this trend, becoming ever more sensitive to the value of the $n_{3 \sigma}$ cut at a fixed threshold of $\Delta \chi^{2} \geqslant 300$. Again, this is expected, as the timescale distributions for the lower and lower masses are typically shorter and shorter compared to the cadence of 15 minutes.

Thus, we find that there is an important and complex interplay between both these criteria, which makes predicting the impact of changes in yield at different values of the photometric precision at a given magnitude more difficult than if we just imposed the $\Delta \chi^{2}$ threshold. As a concrete example to illustrate this point, imagine an event with five data points $8 \sigma$ above the baseline flux and the next most significant point being only $3 \sigma$ above baseline. Further assume that all these points are consecutive and together yield a total $\Delta \chi^{2}=329$. We could change our threshold to $\Delta \chi^{2} \geqslant 329$, and our event would still be counted as detected (at it still passes the $n_{3 \sigma} \geqslant 6$ cut).

As discussed previously, changing the threshold in $\Delta \chi^{2}$ is equivalent to scaling the photometric precision of the survey as function of magnitude. However, simply scaling the yield with the threshold $\Delta \chi^{2}$ does not capture the impact on the $n_{3 \sigma} \geqslant 6$. What is really of interest is the number of detected events when we rescale the individual uncertainties including both criteria. This demonstrates how robust the yield is to degradation or improvement in the photometry. In the above example, assume that the uncertainties are increased by $\sim 4.7 \%$, such that the

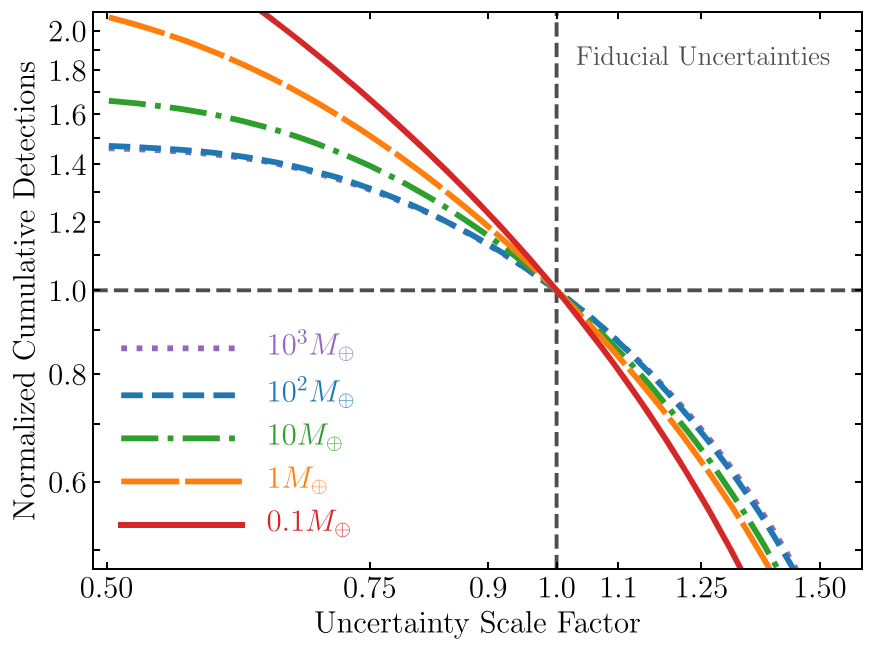

Figure B3. The number of low-mass lens detections that pass both our detection criteria has a higher dependence on the Roman photometric precision than high-mass lenses. These distributions are normalized to the number of events in each mass bin detected when no scaling is applied. The distributions for events with $10^{2}$ and $10^{3} M_{\oplus}$ behave as though only the $\Delta \chi^{2}$ threshold were being scaled, as these events have long timescales and thus are generally robust to the $n_{3 \sigma}$ threshold. However, as lens mass decreases, the slopes of the curves for the lower-mass events continue to steepen for higher error scalings. These masses naturally produce shorter-timescale events, which are much more susceptible to cuts in $n_{3 \sigma}$. Nevertheless, when considering our detection criteria, these distributions show that Roman's yield of low-mass lensing events will degrade gracefully with increasing (fractional) photometric precision.

event now has $\Delta \chi^{2}=300$ and thus would still (barely) pass the $\Delta \chi^{2}$ criterion for detection. However, the last point would now have a significance of $\sim 2.9 \sigma$, and thus the event would fail our $n_{3 \sigma}$ criterion and no longer be detected. This means that those distributions in Figures B1 and B2 can only be used to predict the change in the expected number of detections resulting from changes in the two detection criteria, but they cannot be used to assess the impact on the yield if the photometric precision changes at fixed magnitude. The latter is of more interest when estimating the changes in the survey yield as a result of changes in the mission design.

Thus, to further investigate this interplay, we ran a separate set of modified simulations where everything is the same as described in Section 2, except that we added two calculations. For every event, we calculated the factor by which the uncertainties would need to be uniformly scaled by in order that the total $\Delta \chi^{2}$ of the event is equal to 300 , specifically $C_{\mathrm{DC} 2}=\left(\Delta \chi_{\text {True }}^{2} / 300\right)^{1 / 2}$. We then find the data point that would be the last to qualify the event for the $n_{3 \sigma} \geqslant 6$ cut and calculate the factor to which the photometric uncertainty of that data point would need to be scaled to reach a $3 \sigma$ significance, $C_{\mathrm{N} 3 \mathrm{~S}}$. The lesser of these two factors is the more stringent criterion, and we can therefore assess how the yield changes when the photometric uncertainty is changed, including the impact of both criteria. We find the cumulative distribution of detections as a function of the minimum of these two scaling factors and call it the "Uncertainty Scale Factor" $=\min \left(C_{\mathrm{DC} 2}, C_{\mathrm{N} 3 \mathrm{~S}}\right)$. We plot this distribution normalized to the number of detections expected when the error bars are not scaled at all for our five reference masses in Figure B3.

As one would expect, the higher-mass lenses $\left(10^{3}\right.$ and $10^{2} M_{\oplus}$ ) have essentially the same dependence on this scaling factor. These events typically last longer, so the $n_{3 \sigma}=6$ criterion is generally not approached. For both these masses, 
the behavior is thus the same and is similar to just scaling $\Delta \chi^{2}$. As the mass of lenses drops, the distributions begin to increasingly steepen from 10 to 1 to $0.1 M_{\oplus}$ (green dotteddashed, orange long-dashed, and red solid lines, respectively). As events become shorter and shorter with decreasing mass, more events go undetected owing to the consideration of $n_{3 \sigma}$ when the uncertainties are increased. Fortunately, the inverse is also true. In fact, a larger fraction of events are recovered when the uncertainties are smaller than expected (the spread is larger between the distributions for scale factors less than unity).

The most important takeaway from Figure B3 is that the number of detections is fairly robust to the precise Roman photometric uncertainties that are achieved across a broad range in lens masses, and there are no "thresholds" in the photometric precision below which the detection rate drops precipitously. As a concrete example, for the mostly highly impacted lens mass $0.1 M_{\oplus}$, if precision is $10 \%$ greater than expected, we still recover $\sim 80 \%$ of events.

\section{ORCID iDs}

Samson A. Johnson (iD https://orcid.org/0000-0001-9397-4768 Matthew Penny (i) https://orcid.org/0000-0001-7506-5640 B. Scott Gaudi (i) https://orcid.org/0000-0003-0395-9869 Nicholas J. Rattenbury (iD https://orcid.org/0000-00015069-319X

Annie C. Robin (ib https://orcid.org/0000-0001-8654-9499 Sebastiano Calchi Novati (ib https://orcid.org/0000-00027669-1069

Calen B. Henderson (1D https://orcid.org/0000-0001$8877-9060$

\section{References}

Adams, A. D., Boyajian, T. S., \& von Braun, K. 2018, MNRAS, 473, 3608 Adams, F. C., Anderson, K. R., \& Bloch, A. M. 2013, MNRAS, 432, 438 Agol, E. 2003, ApJ, 594, 449

Akeson, R., Armus, L., Bachelet, E., et al. 2019, arXiv:1902.05569

Alcock, C., Allsman, R. A., Alves, D., et al. 1996, ApJ, 471, 774

Alcock, C., Allsman, R. A., Alves, D., et al. 1997, ApJ, 486, 697

Astropy Collaboration, Price-Whelan, A. M., Sipőcz, B. M., et al. 2018, AJ, 156,123

Astropy Collaboration, Robitaille, T. P., Tollerud, E. J., et al. 2013, A\&A, 558, A33

Bachelet, E., \& Penny, M. 2019, ApJL, 880, L32

Bailey, V. P., Armus, L., Balasubramanian, B., et al. 2019, arXiv:1901.04050 Ban, M. 2020, MNRAS, 494, 3235

Ban, M., Kerins, E., \& Robin, A. C. 2016, A\&A, 595, A53

Barclay, T., Quintana, E. V., Raymond, S. N., \& Penny, M. T. 2017, ApJ, 841,86

Bardalez Gagliuffi, D. C., Burgasser, A. J., Schmidt, S. J., et al. 2019, ApJ, 883, 205

Bate, M. R. 2009, MNRAS, 392, 590

Batygin, K., \& Brown, M. E. 2016, AJ, 151, 22

Bennett, D. P., \& Rhie, S. H. 2002, ApJ, 574, 985

Bennett, D. P., Sumi, T., Bond, I. A., et al. 2012, ApJ, 757, 119

Bonnell, I. A., Clark, P., \& Bate, M. R. 2008, MNRAS, 389, 1556

Borucki, W. J., Koch, D., Basri, G., et al. 2010, Sci, 327, 977

Bozza, V. 2010, MNRAS, 408, 2188

Bozza, V., Bachelet, E., Bartolić, F., et al. 2018, MNRAS, 479, 5157

Cassan, A., Kubas, D., Beaulieu, J.-P., et al. 2012, Natur, 481, 167

Chung, S.-J., Zhu, W., Udalski, A., et al. 2017, ApJ, 838, 154

Claret, A., \& Bloemen, S. 2011, A\&A, 529, A75

Collier Cameron, A., Guenther, E., Smalley, B., et al. 2010, MNRAS, 407, 507

Cushing, M. C., Kirkpatrick, J. D., Gelino, C. R., et al. 2011, ApJ, 743, 50

Dalton, G. B., Caldwell, M., Ward, A. K., et al. 2006, Proc. SPIE, 6269, $62690 X$

Debes, J. H., Ygouf, M., Choquet, E., et al. 2016, JATIS, 2, 011010

Di Stefano, R., \& Scalzo, R. A. 1999, ApJ, 512, 564
Dong, S., Udalski, A., Gould, A., et al. 2007, ApJ, 664, 862 Doyle, L. R., Carter, J. A., Fabrycky, D. C., et al. 2011, Sci, 333, 1602 Flaugher, B., Diehl, H. T., Honscheid, K., et al. 2015, AJ, 150, 150 Gagné, J., Faherty, J. K., Mamajek, E. E., et al. 2017, ApJS, 228, 18 Gaudi, B. S. 2012, ARA\&A, 50, 411

Gaudi, B. S., Akeson, R., Anderson, J., et al. 2019, BAAS, 51, 211

Gaudi, B. S., Stassun, K. G., Collins, K. A., et al. 2017, Natur, 546, 514 Gillon, M., Triaud, A. H. M. J., Demory, B.-O., et al. 2017, Natur, 542, 456 Godines, D., Bachelet, E., Narayan, G., et al. 2019, A\&C, 28, 100298

Gould, A. 1994, ApJL, 421, L75

Gould, A. 1995, ApJL, 441, L21

Gould, A. 1999, ApJ, 514, 869

Gould, A., \& Gaucherel, C. 1997, ApJ, 477, 580

Gould, A., \& Loeb, A. 1992, ApJ, 396, 104

Gould, A., \& Welch, D. L. 1996, ApJ, 464, 212

Griest, K. 1991, ApJ, 366, 412

Griest, K., Cieplak, A. M., \& Lehner, M. J. 2014, ApJ, 786, 158

Han, C., Gaudi, B. S., An, J. H., \& Gould, A. 2005, ApJ, 618, 962

Han, C., \& Kang, Y. W. 2003, ApJ, 596, 1320

Han, C., Lee, C.-U., Udalski, A., et al. 2020a, AJ, 159, 134

Han, C., Park, S.-H., \& Jeong, J.-H. 2000, MNRAS, 316, 97

Han, C., Udalsk, A., Gould, A., et al. 2020b, AJ, 159, 91

Hawley, S. L., Davenport, J. R. A., Kowalski, A. F., et al. 2014, ApJ, 797, 121

Henderson, C. B., Poleski, R., Penny, M., et al. 2016, PASP, 128, 124401

Henderson, C. B., \& Shvartzvald, Y. 2016, AJ, 152, 96

Hestroffer, D., \& Magnan, C. 1998, A\&A, 333, 338

Heyrovský, D. 2003, ApJ, 594, 464

Hodapp, K. W., Kerr, T., Varricatt, W., et al. 2018, Proc. SPIE, 10700, $107002 Z$

Hong, Y.-C., Raymond, S. N., Nicholson, P. D., \& Lunine, J. I. 2018, ApJ, 852,85

Hounsell, R., Scolnic, D., Foley, R. J., et al. 2018, ApJ, 867, 23

Hunter, J. D. 2007, CSE, 9, 90

Kerins, E., Robin, A. C., \& Marshall, D. J. 2009, MNRAS, 396, 1202

Khakpash, S., Penny, M., \& Pepper, J. 2019, AJ, 158, 9

Kim, S.-L., Lee, C.-U., Park, B.-G., et al. 2016, JKAS, 49, 37

Kirkpatrick, J. D., Gelino, C. R., Cushing, M. C., et al. 2012, ApJ, 753, 156

Kreidberg, L., Luger, R., \& Bedell, M. 2019, ApJL, 877, L15

Lee, C.-H., Riffeser, A., Seitz, S., et al. 2009, ApJ, 695, 200

Léger, A., Rouan, D., Schneider, J., et al. 2009, A\&A, 506, 287

Liebes, S. 1964, PhRv, 133, 835

LSST Science Collaboration, Abell, P. A., Allison, J., et al. 2009 arXiv:0912.0201

Luhman, K. L. 2012, ARA\&A, 50, 65

Ma, S., Mao, S., Ida, S., et al. 2016, MNRAS, 461, L107

Malmberg, D., Davies, M. B., \& Heggie, D. C. 2011, MNRAS, 411, 859

Mao, S., \& Paczynski, B. 1991, ApJL, 374, L37

Mayor, M., \& Queloz, D. 1995, Natur, 378, 355

Miyazaki, S., Komiyama, Y., Nakaya, H., et al. 2012, Proc. SPIE, 8446 $84460 Z$

Moniez, M. 2010, GReGr, 42, 2047

Montet, B. T., Yee, J. C., \& Penny, M. T. 2017, PASP, 129, 044401

Mróz, P., Poleski, R., Han, C., et al. 2020, AJ, 159, 262

Mróz, P., Ryu, Y.-H., Skowron, J., et al. 2018, AJ, 155, 121

Mróz, P., Udalski, A., Bennett, D., et al. 2019a, A\&A, 622, A201

Mróz, P., Udalski, A., Skowron, J., et al. 2017, Natur, 548, 183

Mróz, P., Udalski, A., Skowron, J., et al. 2019b, ApJS, 244, 29

Muraki, Y., Sumi, T., Abe, F., et al. 1999, PThPS, 133, 233

Nielsen, E. L., De Rosa, R. J., Macintosh, B., et al. 2019, AJ, 158, 13

Niikura, H., Takada, M., Yasuda, N., et al. 2019, NatAs, 3, 524

Niikura, H., Takada, M., Yokoyama, S., et al. 2019, PhRvD, 99, 083503

Paczyński, B. 1986, ApJ, 304, 1

Paczyński, B. 1991, ApJL, 371, L63

Penny, M., Bachelet, E., Johnson, S., et al. 2019, BAAS, 51, 563

Penny, M. T., Gaudi, B. S., Kerins, E., et al. 2019, ApJS, 241, 3

Penny, M. T., Kerins, E., Rattenbury, N., et al. 2013, MNRAS, 434, 2

Penny, M. T., Rattenbury, N. J., Gaudi, B. S., \& Kerins, E. 2017, AJ, 153, 161

Pfyffer, S., Alibert, Y., Benz, W., et al. 2015, A\&A, 579, A37

Poleski, R., \& Yee, J. 2018, MulensModel: Microlensing Light Curves Modeling, version 1.14.0, Astrophysics Source Code Library, ascl:1803.006

Rasio, F. A., \& Ford, E. B. 1996, Sci, 274, 954

Renault, C., Afonso, C., Aubourg, E., et al. 1997, A\&A, 324, L69

Robin, A. C., Marshall, D. J., Schultheis, M., \& Reylé, C. 2012, A\&A, 538, A106

Robin, A. C., Reylé, C., Derrière, S., \& Picaud, S. 2003, A\&A, 409, 523

Shvartzvald, Y., Bryden, G., Gould, A., et al. 2017, AJ, 153, 61 
Skowron, J., Udalski, A., Gould, A., et al. 2011, ApJ, 738, 87 Specht, D., Kerins, E., Awiphan, S., et al. 2020, arXiv:2005.14668 Spergel, D., Gehrels, N., Baltay, C., et al. 2015, arXiv:1503.03757 Spiegel, D. S., \& Burrows, A. 2012, ApJ, 745, 174

Strigari, L. E., Barnabè, M., Marshall, P. J., et al. 2012, MNRAS, 423, 1856

Sugiyama, S., Kurita, T., \& Takada, M. 2020, MNRAS, 493, 3632

Sumi, T., Bennett, D. P., Bond, I. A., et al. 2013, ApJ, 778, 150

Sumi, T., Kamiya, K., Bennett, D. P., et al. 2011, Natur, 473, 349

Sumi, T., \& Penny, M. T. 2016, ApJ, 827, 139

Suzuki, D., Bennett, D. P., Sumi, T., et al. 2016, ApJ, 833, 145

Takahashi, R., \& Nakamura, T. 2003, ApJ, 595, 1039

Teachey, A., Kipping, D., Burke, C. J., et al. 2020, AJ, 159, 142

Teachey, A., \& Kipping, D. M. 2018, SciA, 4, eaav1784

Troxel, M. A., Long, H., Hirata, C. M., et al. 2019, arXiv:1912.09481

Trujillo, C. A., \& Sheppard, S. S. 2014, Natur, 507, 471

Tsiganis, K., Gomes, R., Morbidelli, A., et al. 2005, Natur, 435, 459

Udalski, A., Szymanski, M., Kaluzny, J., et al. 1992, AcA, 42, 253 van Belle, G. T. 1999, PASP, 111, 1515

van Elteren, A., Portegies Zwart, S., Pelupessy, I., et al. 2019, A\&A, 624, A120

Vietri, M., \& Ostriker, J. P. 1983, ApJ, 267, 488

Witt, H. J. 1995, ApJ, 449, 42

Witt, H. J., \& Mao, S. 1994, ApJ, 430, 505

Wolszczan, A., \& Frail, D. A. 1992, Natur, 355, 145

Woźniak, P., \& Paczyński, B. 1997, ApJ, 487, 55

Wright, E. L., Eisenhardt, P. R. M., Mainzer, A. K., et al. 2010, AJ, 140, 1868

Wyrzykowski, Ł., Rynkiewicz, A. E., Skowron, J., et al. 2015, ApJS, 216, 12

Yee, J. C., Anderson, J., Akeson, R., et al. 2018, arXiv:1803.07921

Yee, J. C., Udalski, A., Calchi Novati, S., et al. 2015, ApJ, 802, 76

Yoo, J., DePoy, D. L., Gal-Yam, A., et al. 2004, ApJ, 603, 139

Zang, W., Penny, M. T., Zhu, W., et al. 2018, PASP, 130, 104401

Zhu, W., \& Gould, A. 2016, JKAS, 49, 93

Zhu, W., Huang, C. X., Udalski, A., et al. 2017a, PASP, 129, 104501

Zhu, W., Udalski, A., Huang, C. X., et al. 2017b, ApJL, 849, L31 